\title{
Subsubleading soft theorems of gravitons and dilatons in the bosonic string
}

\author{
Paolo Di Vecchia, ${ }^{a, b}$ Raffaele Marotta $^{c}$ and Matin Mojaza ${ }^{b}$ \\ ${ }^{a}$ The Niels Bohr Institute, University of Copenhagen, \\ Blegdamsvej 17, DK-2100 Copenhagen Ø, Denmark \\ ${ }^{b}$ NORDITA, KTH Royal Institute of Technology and Stockholm University, \\ Roslagstullsbacken 23, SE-10691 Stockholm, Sweden \\ ${ }^{c}$ Istituto Nazionale di Fisica Nucleare, Sezione di Napoli, \\ Complesso Universitario di Monte S. Angelo ed. 6, via Cintia, 80126, Napoli, Italy \\ E-mail: divecchi@nbi.dk, raffaele.marotta@na.infn.it, \\ mojaza@nordita.org
}

ABSTRACT: Starting from the amplitude with an arbitrary number of massless closed states of the bosonic string, we compute the soft limit when one of the states becomes soft to subsubleading order in the soft momentum expansion, and we show that when the soft state is a graviton or a dilaton, the full string amplitude can be expressed as a soft theorem through subsubleading order. It turns out that there are string corrections to the field theoretical limit in the case of a soft graviton, while for a soft dilaton the string corrections vanish. We then show that the new soft theorems, including the string corrections, can be simply obtained from the exchange diagrams where the soft state is attached to the other external states through the three-point string vertex of three massless states. In the soft-limit, the propagator of the exchanged state is divergent, and at tree-level these are the only divergent contributions to the full amplitude. However, they do not form a gauge invariant subset and must be supplemented with extra non-singular terms. The requirement of gauge invariance then fixes the complete amplitude through subsubleading order in the soft expansion, reproducing exactly what one gets from the explicit calculation in string theory. From this it is seen that the string corrections at subsubleading order arise as a consequence of the three-point amplitude having string corrections in the bosonic string. When specialized to a soft dilaton, it remarkably turns out that the string corrections vanish and that the non-singular piece of the subsubleading term of the dilaton soft theorem is the generator of space-time special conformal transformation.

Keywords: Bosonic Strings, Scattering Amplitudes

ARXiv EPrint: 1604.03355 


\section{Contents}

1 Introduction and summary of results 1

$2 \quad$ Amplitude of one soft and $n$ massless closed strings 4

$\begin{array}{lll}3 & \text { Soft gravitons and dilatons } & 10\end{array}$

4 String corrections from gauge invariance $\quad 13$

$\begin{array}{lll}5 & \text { Discussion and conclusion } & 17\end{array}$

$\begin{array}{lr}\text { A Results of integrals } & 18\end{array}$

B Matching with the subsubleading soft operator 23

\section{Introduction and summary of results}

Tremendous progress is happening in understanding the soft factorizing behavior of scattering amplitudes and their relation to underlying, sometimes hidden, symmetries. Most remarkable, perhaps, are the suggestions that the soft behavior of particularly the gravity and Yang-Mills S-matrices are related to asymptotic symmetries in general relativity and in gauge theories [1-22]. Also remarkable are the similarities pointed out very recently between the soft behavior of the gravity/string dilaton and the Ward identities of scale and special conformal transformations [23, 24]. New uses of soft theorems are also being discovered in the more modern field of amplitudes [25, 26].

Soft theorems, however, have a long history, and go back to the seminal works in the 1950s on low-energy photon scattering [27-33] and in the 1960s on soft-graviton scattering [34-37], when they were realized to be important consequences of gauge invariance. Discussions on the generic subleading behavior of soft gluon and graviton scattering were recently taken up in [38-40], and has, since the suggested relations to asymptotic symmetries [1-22], received enormous attention, not only in gravity and Yang-Mills theory [41-63], but also their extensions in supersymmetric theories [64-72], and in string theory [73-78]. Double-soft theorems are also receiving increasing interest [79-84], due to their potential to uncover hidden symmetries of the S-matrix (see e.g. ref. [85] for a discussion on Adler's zeroes and the pion double-soft theorem).

Soft theorems in string theory were first discussed in the 1970s by Ademollo et al. [86] and by Shapiro [87] for tree diagram scattering amplitudes involving massless particles only, and with particular emphasis on the string-dilaton as the soft-state (see also refs. [88, 89] for this study in string field theory). In a recent work [78], we have revived this line of studies by computing the soft behavior up to subsubleading order, when a soft massless closed string-state is scattering on external tachyons. It turns out that this amplitude is 
determined by the same gauge invariance that also determines the soft-graviton behavior up to subsubleading order in field theory, derived in ref. [62]. Furthermore, we computed the leading soft-behavior of the antisymmetric Kalb-Ramond tensor in the scattering on other massless closed string states. At the same time we rederived the known results involving instead a soft graviton or dilaton, and showed by invoking a slight generalization of the analysis done in ref. [62] that the leading soft behavior for both of them is again determined by field theory gauge invariance. The aim of this work is to extend our previous analysis in the bosonic string to the subsubleading order for the case of a soft graviton or dilaton scattering on other closed massless states. At this order, string corrections to the corresponding field theory soft theorems are expected to appear for the first time [63], and indeed this is what we find. Their presence is also expected in heterotic string and it is due to interaction terms of the type $\phi R^{2}$ which appear, to order $\alpha^{\prime}$, in the effective actions of such string theories [90]. String corrections to the graviton soft theorem have also been computed in [91] in the case of four point bosonic string amplitudes. We have extended this analysis to $n+1$ point amplitudes with a soft dilaton or graviton and $n$ massless hard particles finding that only the graviton soft operator is modified by $\alpha^{\prime}$-corrections. The lack of string corrections in the soft behavior of the dilaton could be a signal that the dilaton soft theorem is a consequence of some Ward identity as it occurs for the NambuGoldstone boson of the spontaneously broken conformal invariance [24]. The similarities between these two particles, both called dilaton, indeed deserve a further study.

Let us summarize our primary results before going through the calculational details.

In ref. [24] it has been shown that the field theory amplitude for a soft graviton or a soft dilaton of soft momentum $q$, with $n$ other hard gravitons and/or dilatons can be written in the following factorized form:

$$
M_{n+1}\left(q ; k_{i}\right) \equiv \epsilon_{\mu \nu}^{S} M^{\mu \nu}\left(k_{i} ; q\right)=\kappa_{D}\left(\hat{S}_{q}^{(-1)}+\hat{S}_{q}^{(0)}+\hat{S}_{q}^{(1)}\right) M_{n}\left(k_{i}\right)+\mathcal{O}\left(q^{2}\right),
$$

where $\kappa_{D}$ is related to the gravitational constant in $D$ space-time dimension, the superscript of each $\hat{S}_{q}^{(m)}$ indicates the order $m$ in $q$ of each term, and $M_{n}$ is the amplitude without the soft particle. $\epsilon_{\mu \nu}^{S}$ is the polarization of either the graviton or the dilaton, which is symmetric under the exchange of $\mu$ and $\nu$. In ref. [78], the above soft theorem to subleading order was shown to hold also in the framework of the bosonic string including also the Kalb-Ramond antisymmetric field both in the role of the soft state and as hard states.

In the cases of a soft graviton or dilaton, the first two terms are given by [78]:

$$
\hat{S}_{q}^{(-1)}=\epsilon_{\mu \nu}^{S} \sum_{i=1}^{n} \frac{k_{i}^{\mu} k_{i}^{\nu}}{k_{i} \cdot q}, \quad \hat{S}_{q}^{(0)}=\epsilon_{\mu \nu}^{S}\left(-\frac{i q_{\rho}}{2}\right) \sum_{i=1}^{n} \frac{k_{i}^{\mu} J_{i}^{\nu \rho}+k_{i}^{\nu} J_{i}^{\mu \rho}}{k_{i} \cdot q}
$$

where

$$
\begin{aligned}
J_{i}^{\mu \nu} & =L_{i}^{\mu \nu}+\mathcal{S}_{i}^{\mu \nu}, & \mathcal{S}_{i}^{\mu \nu} & =S_{i}^{\mu \nu}+\bar{S}_{i}^{\mu \nu}, \\
L_{i}^{\mu \nu} & =i\left(k_{i}^{\mu} \frac{\partial}{\partial k_{i \nu}}-k_{i}^{\nu} \frac{\partial}{\partial k_{i \mu}}\right), & S_{i}^{\mu \nu} & =i\left(\epsilon_{i}^{\mu} \frac{\partial}{\partial \epsilon_{i \nu}}-\epsilon_{i}^{\nu} \frac{\partial}{\partial \epsilon_{i \mu}}\right), \quad \bar{S}_{i}^{\mu \nu}=i\left(\bar{\epsilon}_{i}^{\mu} \frac{\partial}{\partial \bar{\epsilon}_{i \nu}}-\bar{\epsilon}_{i}^{\nu} \frac{\partial}{\partial \bar{\epsilon}_{i \mu}}\right) .
\end{aligned}
$$


while the third term was computed in the field theory limit in ref. [24]. The method used is an extension of the one of ref. [62] and the soft behavior in eq. (1.1) is shown to be a direct consequence of the gauge invariance conditions

$$
q_{\mu} M^{\mu \nu}\left(k_{i} ; q\right)=q_{\nu} M^{\mu \nu}\left(k_{i} ; q\right)=0 .
$$

In this paper we extend the previous method to include string corrections and we check the final result by performing a direct calculation of the subsubleading term in the soft limit in the amplitude of the bosonic string involving an arbitrary number of massless closed strings. This calculation is performed by extending the technique developed in ref. [78] for the computation of the subleading term. As a result we obtain the following subsubleading term:

$$
\begin{aligned}
S_{q}^{(1)}= & -\frac{\epsilon_{\mu \nu}^{S}}{2} \sum_{i=1}^{n}\left[\frac{q_{\rho} J_{i}^{\mu \rho} q_{\sigma} J_{i}^{\nu \sigma}}{k_{i} \cdot q}+\left(\frac{k_{i}^{\mu} q^{\nu}}{k_{i} \cdot q} q^{\sigma}+q^{\mu} \eta^{\nu \sigma}-\eta^{\mu \nu} q^{\sigma}\right) \frac{\partial}{\partial k_{i}^{\sigma}}\right. \\
& -\left(\frac{q_{\rho} q_{\sigma} \eta_{\mu \nu}-q_{\sigma} q_{\nu} \eta_{\rho \mu}-q_{\rho} q_{\mu} \eta_{\sigma \nu}}{k_{i} \cdot q}\right) \Pi_{i}^{\rho \sigma} \\
& \left.-\alpha^{\prime}\left(q_{\sigma} k_{i \nu} \eta_{\rho \mu}+q_{\rho} k_{i \mu} \eta_{\sigma \nu}-\eta_{\rho \mu} \eta_{\sigma \nu}\left(k_{i} \cdot q\right)-q_{\rho} q_{\sigma} \frac{k_{i \mu} k_{i \nu}}{k_{i} \cdot q}\right) \Pi_{i}^{\rho \sigma}\right]
\end{aligned}
$$

where

$$
\Pi_{i}^{\rho \sigma}=\epsilon_{i}^{\rho} \frac{\partial}{\partial \epsilon_{i \sigma}}+\bar{\epsilon}_{i}^{\rho} \frac{\partial}{\partial \bar{\epsilon}_{i \sigma}} .
$$

Only the symmetric part $\Pi_{i}^{\{\rho, \sigma\}}=\frac{\Pi_{i}^{\rho \sigma}+\Pi_{i}^{\sigma \rho}}{2}$ contributes in the previous expression because the polarization tensor $\epsilon_{\mu \nu}^{S}$ is symmetric in the indices $\mu$ and $\nu$. The first two lines of eq. (1.6) agree with the expression already presented in ref. [24], while the third line gives the string corrections.

By choosing the polarization of the graviton, from eqs. (1.2) and (1.6) we get the soft theorem of a graviton:

$$
\begin{aligned}
M_{n+1}^{\text {graviton }}= & \kappa_{D} \epsilon_{\mu \nu}^{g} \sum_{i=1}^{n}\left[\frac{k_{i}^{\mu} k_{i}^{\nu}-i q_{\rho} k_{i}^{\mu} J_{i}^{\nu \rho}-\frac{1}{2} q_{\rho} J_{i}^{\mu \rho} q_{\sigma} J_{i}^{\nu \sigma}}{k_{i} q}\right. \\
& \left.-\frac{\alpha^{\prime}}{2}\left(q_{\sigma} k_{i \nu} \eta_{\rho \mu}+q_{\rho} k_{i \mu} \eta_{\sigma \nu}-\eta_{\rho \mu} \eta_{\sigma \nu}\left(k_{i} \cdot q\right)-q_{\rho} q_{\sigma} \frac{k_{i \mu} k_{i \nu}}{k_{i} \cdot q}\right) \Pi_{i}^{\{\rho, \sigma\}}\right] M_{n},
\end{aligned}
$$

while, by choosing the polarization tensor of the dilaton $\epsilon_{\mu \nu}^{S} \rightarrow \frac{1}{\sqrt{D-2}}\left(\eta_{\mu \nu}-q_{\mu} \bar{q}_{\nu}-q_{\nu} \bar{q}_{\mu}\right)$, we get the soft theorem for a dilaton:

$$
\begin{aligned}
M_{n+1}^{\text {dilaton }}= & \frac{\kappa_{D}}{\sqrt{D-2}}\left[2-\sum_{i=1}^{n} k_{i \mu} \frac{\partial}{\partial k_{i \mu}}\right. \\
& \left.+\frac{1}{2} \sum_{i=1}^{n}\left(q^{\rho} \hat{K}_{i \rho}+\frac{q^{\rho} q^{\sigma}}{k_{i} q}\left(\mathcal{S}_{i, \rho \mu} \eta^{\mu \nu} \mathcal{S}_{i \nu \sigma}+D \Pi_{i,\{\rho, \sigma\}}\right)\right)\right] M_{n}
\end{aligned}
$$

where

$$
\hat{K}_{i \mu}=2\left[\frac{1}{2} k_{i \mu} \frac{\partial^{2}}{\partial k_{i \nu} \partial k_{i}^{\nu}}-k_{i}^{\rho} \frac{\partial^{2}}{\partial k_{i}^{\mu} \partial k_{i}^{\rho}}+i \mathcal{S}_{i, \rho \mu} \frac{\partial}{\partial k_{i}^{\rho}}\right]
$$


Remarkably, these operators are nothing but the generators of space-time special conformal transformations acting in momentum space. As recently shown in ref. [24], these operators also control the soft behavior of the Nambu-Goldstone bosons of spontaneously broken conformal invariance and an interesting application of this recently appeared in ref. [26]. It would be interesting to understand the physical reason for why these generators appear in the soft limit of the string dilaton. Notice furthermore that the string corrections vanish completely for a soft dilaton.

The paper is organized as follows. In section 2 we write the amplitude with an arbitrary number of massless states of the closed bosonic string and we perform the limit in which one of them (a dilaton or a graviton) becomes soft. In section 3 we derive the explicit form of the soft behavior for a dilaton and for a graviton and we give a physical interpretation of the various terms that appear. In section 4 we derive the string corrections to the soft theorem through gauge invariance from the string corrections of the three-point amplitude for massless closed string states. Finally, details of the calculations presented in section 2 are given in two appendices.

\section{$2 \quad$ Amplitude of one soft and $n$ massless closed strings}

In this section we consider the amplitude with $n+1$ massless closed string states and we study its behavior in the limit in which one of the massless states is soft. We start by summarizing the results presented in ref. [78], where more details may be found.

The amplitude involving $n+1$ massless closed string states can be written as

$$
\begin{aligned}
M_{n+1} \sim & \int \frac{\prod_{i=1}^{n} d^{2} z_{i} d^{2} z}{d V_{a b c}} \int d \theta \prod_{i=1}^{n} d \theta_{i}\left\langle 0\left|e^{i\left(\theta \epsilon_{q}^{\mu} \partial_{z}+\sqrt{\frac{\alpha^{\prime}}{2}} q^{\mu}\right) X_{\mu}(z)} \prod_{i=1}^{n} e^{i\left(\theta_{i} \epsilon_{i}^{\mu_{i}} \partial_{z_{i}}+\sqrt{\frac{\alpha^{\prime}}{2}} k_{i}^{\mu_{i}}\right) X_{\mu_{i}}\left(z_{i}\right)}\right| 0\right\rangle \\
& \times \int d \bar{\theta} \prod_{i=1}^{n} d \bar{\theta}_{i}\left\langle 0\left|e^{i\left(\bar{\theta} \bar{\epsilon}_{q}^{\mu} \partial_{\bar{z}}+\sqrt{\frac{\alpha^{\prime}}{2}} q^{\mu}\right) X_{\mu}(\bar{z})} \prod_{i=1}^{n} e^{i\left(\bar{\theta}_{i} \bar{\epsilon}_{i}^{\nu_{i}} \partial_{\bar{z}_{i}}+\sqrt{\frac{\alpha^{\prime}}{2}} k_{i}^{\nu_{i}}\right) X_{\nu_{i}}\left(\bar{z}_{i}\right)}\right| 0\right\rangle
\end{aligned}
$$

where we assume that $\theta, \bar{\theta}, \epsilon, \bar{\epsilon}$ are Grassmann variables, and we use the definition $\epsilon_{i \mu \nu} \equiv \epsilon_{i \mu} \bar{\epsilon}_{i \nu}$ for the polarization tensor. We consider the soft string to be the one with momentum $q$ and polarization $\epsilon_{q, \mu \nu}$. After using the contraction $\left\langle X^{\mu}(z) X^{\nu}(w)\right\rangle=$ $-\eta^{\mu \nu} \log (z-w)$ and performing the integration over the Grassmann variables $\theta$ and $\bar{\theta}$, the expression reduces to a form which can formally be written in two parts:

$$
M_{n+1}=M_{n} * S,
$$

where by $*$ a convolution of integrals is understood, and where

$$
\begin{aligned}
S \equiv & \kappa_{D} \int \frac{d^{2} z}{2 \pi} \sum_{i=1}^{n}\left(\theta_{i} \frac{\left(\epsilon_{q} \epsilon_{i}\right)}{\left(z-z_{i}\right)^{2}}+\sqrt{\frac{\alpha^{\prime}}{2}} \frac{\left(\epsilon_{q} k_{i}\right)}{z-z_{i}}\right) \sum_{j=1}^{n}\left(\bar{\theta}_{j} \frac{\left(\bar{\epsilon}_{q} \bar{\epsilon}_{j}\right)}{\left(\bar{z}-\bar{z}_{j}\right)^{2}}+\sqrt{\frac{\alpha^{\prime}}{2}} \frac{\left(\bar{\epsilon}_{q} k_{i}\right)}{\bar{z}-\bar{z}_{i}}\right) \\
& \times \exp \left[-\sqrt{\frac{\alpha^{\prime}}{2}} \sum_{i=1}^{n} \theta_{i} \frac{\left(\epsilon_{i} q\right)}{z-z_{i}}\right] \exp \left[-\sqrt{\frac{\alpha^{\prime}}{2}} \sum_{i=1}^{n} \bar{\theta}_{i} \frac{\left(\bar{\epsilon}_{i} q\right)}{\bar{z}-\bar{z}_{i}}\right] \prod_{i=1}^{n}\left|z-z_{i}\right|^{\alpha^{\prime} q k_{i}},
\end{aligned}
$$


is the part describing the soft particle, and

$$
\begin{aligned}
M_{n}= & \frac{8 \pi}{\alpha^{\prime}}\left(\frac{\kappa_{D}}{2 \pi}\right)^{n-2} \int \frac{\prod_{i=1}^{n} d^{2} z_{i}}{d V_{a b c}} \int\left[\prod_{i=1}^{n} d \theta_{i} \prod_{i=1}^{n} d \bar{\theta}_{i}\right] \prod_{i<j}\left|z_{i}-z_{j}\right|^{\alpha^{\prime} k_{i} k_{j}} \\
& \times \exp \left[-\sum_{i<j} \frac{\theta_{i} \theta_{j}}{\left(z_{i}-z_{j}\right)^{2}}\left(\epsilon_{i} \epsilon_{j}\right)+\sqrt{\frac{\alpha^{\prime}}{2}} \sum_{i \neq j} \frac{\theta_{i}\left(\epsilon_{i} k_{j}\right)}{z_{i}-z_{j}}\right] \\
& \times \exp \left[-\sum_{i<j} \frac{\bar{\theta}_{i} \bar{\theta}_{j}}{\left(\bar{z}_{i}-\bar{z}_{j}\right)^{2}}\left(\bar{\epsilon}_{i} \bar{\epsilon}_{j}\right)+\sqrt{\frac{\alpha^{\prime}}{2}} \sum_{i \neq j} \frac{\bar{\theta}_{i}\left(\bar{\epsilon}_{i} k_{j}\right)}{\bar{z}_{i}-\bar{z}_{j}}\right]
\end{aligned}
$$

is the amplitude of $n$ massless states without the soft particle.

We eventually want to find a soft operator $\hat{S}$ such that $\hat{S} M_{n}=M_{n} * S$ through order $q^{1}$. This can be done by expanding $S$ for small $q$ and keep terms in the integrand up to the order $q^{2}$, since higher orders of the integrand cannot yield terms of order $q^{1}$ after integration. It is useful then to divide $S$ in three parts:

$$
S=\kappa_{D}\left(S_{1}+S_{2}+S_{3}\right)+\mathcal{O}\left(q^{2}\right)
$$

defined by:

$$
\begin{aligned}
S_{1}= & \frac{\alpha^{\prime}}{2} \int \frac{d^{2} z}{2 \pi} \sum_{i=1}^{n} \frac{\left(\epsilon_{q} k_{i}\right)}{z-z_{i}} \sum_{j=1}^{n} \frac{\left(\bar{\epsilon}_{q} k_{j}\right)}{\bar{z}-\bar{z}_{j}} \prod_{i=1}^{n}\left|z-z_{i}\right|^{\alpha^{\prime} q k_{i}} \\
& \times\left\{1-\sqrt{\frac{\alpha^{\prime}}{2}} \sum_{k=1}^{n}\left(\theta_{k} \frac{\left(\epsilon_{k} q\right)}{z-z_{k}}+\bar{\theta}_{k} \frac{\left(\bar{\epsilon}_{k} q\right)}{\bar{z}-\bar{z}_{k}}\right)+\frac{1}{2}\left(\frac{\alpha^{\prime}}{2}\right)\right. \\
& \left.\times\left[\left(\sum_{h=1}^{n} \theta_{h} \frac{\left(\epsilon_{h} q\right)}{z-z_{h}}\right)^{2}+\left(\sum_{h=1}^{n} \bar{\theta}_{h} \frac{\left(\bar{\epsilon}_{h} q\right)}{\bar{z}-\bar{z}_{h}}\right)^{2}+2\left(\sum_{h=1}^{n} \theta_{h} \frac{\left(\epsilon_{h} q\right)}{z-z_{h}}\right)\left(\sum_{h=1}^{n} \bar{\theta}_{h} \frac{\left(\bar{\epsilon}_{h} q\right)}{\bar{z}-\bar{z}_{h}}\right)\right]\right\} \\
S_{2}= & \int \frac{d^{2} z}{2 \pi} \sum_{i=1}^{n}\left(\theta_{i} \frac{\left(\epsilon_{q} \epsilon_{i}\right)}{\left(z-z_{i}\right)^{2}}\right) \sum_{j=1}^{n}\left(\bar{\theta}_{j} \frac{\left(\bar{\epsilon}_{q} \bar{\epsilon}_{j}\right)}{\left(\bar{z}-\bar{z}_{j}\right)^{2}}\right) \prod_{\ell=1}^{n}\left|z-z_{\ell}\right|^{\alpha^{\prime} q k_{\ell}} \\
& \times\left\{1-\sqrt{\frac{\alpha^{\prime}}{2}} \sum_{k=1}^{n}\left(\theta_{k} \frac{\epsilon_{k} q}{z-z_{k}}+\bar{\theta}_{k} \frac{\left(\bar{\epsilon}_{k} q\right)}{\bar{z}-\bar{z}_{k}}\right)^{\prime}+\frac{1}{2}\left(\frac{\alpha^{\prime}}{2}\right)\right. \\
& \left.\times\left[\left(\sum_{h=1}^{n} \theta_{h} \frac{\left(\epsilon_{h} q\right)}{z-z_{h}}\right)^{2}+\left(\sum_{h=1}^{n} \bar{\theta}_{h} \frac{\left(\bar{\epsilon}_{h} q\right)}{\bar{z}-\bar{z}_{h}}\right)^{2}+2\left(\sum_{h=1}^{n} \theta_{h} \frac{\left(\epsilon_{h} q\right)}{z-z_{h}}\right)\left(\sum_{h=1}^{n} \bar{\theta}_{h} \frac{\left(\bar{\epsilon}_{h} q\right)}{\bar{z}-\bar{z}_{h}}\right)\right]\right\} \\
S_{3}= & \sqrt{\frac{\alpha^{\prime}}{2}} \int \frac{d^{2} z}{2 \pi} \sum_{i=1}^{n} \sum_{j=1}^{n}\left[\left(\frac{\theta_{i}\left(\epsilon_{q} \epsilon_{i}\right)}{\left(z-z_{i}\right)^{2}}\right)\left(\frac{\left(\bar{\epsilon}_{q} k_{j}\right)}{\bar{z}^{\prime}-\bar{z}_{j}}\right)+\left(\frac{\bar{\theta}_{i}\left(\bar{\epsilon}_{q} \bar{\epsilon}_{i}\right)}{\left(\bar{z}-\bar{z}_{i}\right)^{2}}\right)\left(\frac{\left(\epsilon_{q} k_{j}\right)}{z-z_{j}}\right)\right] \prod_{\ell=1}^{n}\left|z-z_{\ell}\right|^{\alpha^{\prime} q k_{\ell}} \\
& \times\left\{1-\left(\frac{\sqrt{2 \alpha^{\prime}}}{2}\right) \sum_{k=1}^{n}\left(\theta_{k} \frac{\epsilon_{k} q}{z-z_{k}}+\bar{\theta}_{k} \frac{\left(\bar{\epsilon}_{k} q\right)}{\bar{z}-\bar{z}_{k}}\right)+\frac{1}{2}\left(\frac{\alpha^{\prime}}{2}\right)\right. \\
& \left.\times\left[\left(\sum_{h=1}^{n} \theta_{h} \frac{\left(\epsilon_{h} q\right)}{z-z_{h}}\right)^{2}+\left(\sum_{h=1}^{n} \bar{\theta}_{h} \frac{\left(\bar{\epsilon}_{h} q\right)}{\bar{z}-\bar{z}_{h}}\right)^{2}+2\left(\sum_{h=1}^{n} \theta_{h} \frac{\left(\epsilon_{h} q\right)}{z-z_{h}}\right)\left(\sum_{h=1}^{n} \bar{\theta}_{h} \frac{\left(\bar{\epsilon}_{h} q\right)}{\bar{z}-\bar{z}_{h}}\right)\right]\right\}
\end{aligned}
$$


These terms provide all contributions to the order $q^{1}$. They can be further split in $S_{i}^{(a)}$, $\mathrm{a}=0,1,2$, with the index $a$ labelling the order of expansion in $q$ of the integrand modulo the factor $\left|z-z_{l}\right|^{\alpha^{\prime} q k_{l}}$, which has to be integrated. The integrals involved are all of the form:

$$
I_{i_{1} i_{2} \ldots}^{j_{1} j_{2} \ldots}=\int \frac{d^{2} z}{2 \pi} \frac{\prod_{l=1}^{n}\left|z-z_{l}\right|^{\alpha^{\prime} k_{l} q}}{\left(z-z_{i_{1}}\right)\left(z-z_{i_{2}}\right) \cdots\left(\bar{z}-\bar{z}_{j_{1}}\right)\left(\bar{z}-\bar{z}_{j_{2}}\right) \cdots} .
$$

Each of the integrals involved has to be computed through order $q^{1-a}$, which we denote by $I^{(1-a)}{ }_{i_{1} i_{2} \ldots}^{j_{1} \ldots}$. Using this notation, each term can be compactly expressed as:

$$
\begin{aligned}
& S_{1}^{(0)}=\frac{\alpha^{\prime}}{2} \sum_{i=1}^{n}\left[\left(\epsilon_{q} k_{i}\right)\left(\bar{\epsilon}_{q} k_{i}\right) I_{i}^{(1)^{i}}+\sum_{j \neq i}^{n}\left(\epsilon_{q} k_{i}\right)\left(\bar{\epsilon}_{q} k_{j}\right) I_{i}^{(1)}{ }_{i}^{j}\right] \\
& S_{1}^{(1)}=-\left(\frac{\alpha^{\prime}}{2}\right)^{\frac{3}{2}} \sum_{i, j, l=1}^{n}\left(\epsilon_{q} k_{i}\right)\left(\bar{\epsilon}_{q} k_{j}\right)\left(\theta_{l} \epsilon_{l} q\right) I_{i l}^{(0)^{j}}+\text { c.c. } \\
& S_{1}^{(2)}=\frac{1}{2}\left(\frac{\alpha^{\prime}}{2}\right)^{2} \sum_{i, j, l=1}^{n}\left(\epsilon_{q} k_{i}\right)\left(\bar{\epsilon}_{q} k_{j}\right)\left(\theta_{l} \epsilon_{l} q\right)\left[\sum_{m \neq l}^{n}\left(\theta_{m} \epsilon_{m} q\right) I_{i l m}^{(-1)^{j}}+\sum_{m=1}^{n}\left(\bar{\theta}_{m} \bar{\epsilon}_{m} q\right) I_{i l}^{(-1)}{ }_{i l}^{j m}\right]+\text { c.c. }
\end{aligned}
$$

with c.c. denoting the complex conjugate of the expressions. Similarly:

$$
\begin{aligned}
S_{2}^{(0)}= & \sum_{i, j=1}^{n}\left(\epsilon_{q} \theta_{i} \epsilon_{i}\right)\left(\bar{\epsilon}_{q} \bar{\theta}_{j} \bar{\epsilon}_{j}\right) I_{i i}^{(1)^{j j}}, \\
S_{2}^{(1)}= & -\sqrt{\frac{\alpha^{\prime}}{2}} \sum_{i, j=1}^{n} \sum_{l \neq i=1}^{n}\left(\epsilon_{q} \theta_{i} \epsilon_{i}\right)\left(\bar{\epsilon}_{q} \bar{\theta}_{j} \bar{\epsilon}_{j}\right)\left(\theta_{l} \epsilon_{l} q\right) I^{(0)^{j j}}+\text { c.c. }, \\
S_{2}^{(2)}= & \frac{\alpha^{\prime}}{4} \sum_{i, j=1}^{n}\left(\epsilon_{q} \theta_{i} \epsilon_{i}\right)\left(\bar{\epsilon}_{q} \bar{\theta}_{j} \bar{\epsilon}_{j}\right)\left[\sum_{l \neq m \neq i=1}^{n}\left(\theta_{l} \epsilon_{l} q\right)\left(\theta_{m} \epsilon_{m} q\right) I^{(-1)^{j j} j m}\right. \\
& \left.+\sum_{l \neq i=1}^{n} \sum_{m \neq j=1}^{n}\left(\theta_{l} \epsilon_{l} q\right)\left(\bar{\theta}_{m} \bar{\epsilon}_{m} q\right) I^{(-1)_{i i l}^{j j m}}\right]+ \text { c.c. },
\end{aligned}
$$

where we note that according to eq. (A.38) the first part of $S_{2}^{(2)}$ involving $I_{i i l m}^{j j}$ does not contribute to the order $q$ for any $i, j$ and $l \neq m \neq i$. Finally:

$$
\begin{aligned}
S_{3}^{(0)} & =\sqrt{\frac{\alpha^{\prime}}{2}} \sum_{i, j=1}^{n}\left(\epsilon_{q} \theta_{i} \epsilon_{i}\right)\left(\bar{\epsilon}_{q} k_{j}\right) I^{(1)^{j}}{ }_{i i}+\text { c.c. }, \\
S_{3}^{(1)} & =-\frac{\alpha^{\prime}}{2} \sum_{i, j=1}^{n}\left(\epsilon_{q} \theta_{i} \epsilon_{i}\right)\left(\bar{\epsilon}_{q} k_{j}\right)\left[\sum_{l \neq i=1}^{n}\left(\theta_{l} \epsilon_{l} q\right) I^{(0)^{j}}{ }_{i i l}+\sum_{l=1}^{n}\left(\bar{\theta}_{j} \bar{\epsilon}_{j} q\right) I^{(0)^{j l}}{ }_{i i}\right]+\text { c.c. }, \\
S_{3}^{(2)} & =\frac{1}{2}\left(\frac{\alpha^{\prime}}{2}\right)^{\frac{3}{2}} \sum_{i, j=1}^{n}\left(\epsilon_{q} \theta_{i} \epsilon_{i}\right)\left(\bar{\epsilon}_{q} k_{j}\right)\left[\sum_{l \neq m \neq i=1}^{n}\left(\theta_{l} \epsilon_{l} q\right)\left(\theta_{m} \epsilon_{m} q\right) I^{(-1)^{j}}{ }_{i i l m}\right.
\end{aligned}
$$




$$
\left.+\sum_{l \neq m=1}\left(\bar{\theta}_{l} \bar{\epsilon}_{l} q\right)\left(\bar{\theta}_{m} \bar{\epsilon}_{m} q\right) I^{(-1)_{i i}^{j l m}}+\sum_{l \neq i=1}^{n} \sum_{m \neq j=1}^{n}\left(\theta_{l} \epsilon_{l} q\right)\left(\bar{\theta}_{m} \bar{\epsilon}_{m} q\right) I_{i i l}^{(-1)^{j m}}\right]+ \text { c.c. },
$$

where we note that by inspection of eqs. (A.22)-(A.28) the second part of $S_{3}^{(2)}$ involving $I_{i i}^{j l m}$ does not contribute to the order $q$ for any $j, l$, and $m \neq l$.

As a word of warning, notice that the definitions of $S_{i}$ are not the same as in ref. [78], but one can identify $S_{1}^{(0)}, S_{1}^{(1)}, S_{2}^{(0)}+S_{2}^{(1)}$, and $S_{3}^{(0)}+S_{3}^{(1)}$, with respectively $S_{1}, S_{2}, S_{4}$ and $S_{3}$ of ref. [78]. In appendix A we provide the computational details as well as the explicit results for all the integrals involved. In particular, we show in the appendix that all the integrals are linear combinations of a subset of six of them. The coefficients of these linear combinations are complex functions with poles when two Koba-Nielsen variables coincide. We now report the results.

The first term of $S_{1}$, i.e. $S_{1}^{(0)}$, is the part equivalent to the amplitude of a soft massless string scattering on $n$ tachyons, and this was already computed to the order $q^{1}$ in ref. [78], reading:

$$
\begin{aligned}
S_{1}^{(0)}= & \epsilon_{q}^{S \mu \nu}\left\{\sum _ { i = 1 } ^ { n } k _ { i \mu } k _ { i \nu } \left[\frac{\left(\alpha^{\prime}\right)^{2}}{2} \sum_{j \neq i}\left(k_{j} q\right) \log ^{2}\left|z_{i}-z_{j}\right|\right.\right. \\
& \left.+\frac{1}{k_{i} q}\left(1+\alpha^{\prime} \sum_{j \neq i}\left(k_{j} q\right) \log \left|z_{i}-z_{j}\right|+\frac{\left(\alpha^{\prime}\right)^{2}}{2} \sum_{j \neq i} \sum_{k \neq i}\left(k_{j} q\right)\left(k_{k} q\right) \log \left|z_{i}-z_{j}\right| \log \left|z_{i}-z_{k}\right|\right)\right] \\
& -\alpha^{\prime} \sum_{i \neq j}^{n} k_{i \mu} k_{j \nu}\left[\log \left|z_{i}-z_{j}\right|-\frac{\alpha^{\prime}}{2} \sum_{m \neq i, j}\left(q k_{m}\right) \log \left|z_{m}-z_{j}\right| \log \left|z_{i}-z_{m}\right|\right. \\
& \left.\left.+\frac{\alpha^{\prime}}{2} \sum_{m \neq j}\left(q k_{m}\right) \log \left|z_{m}-z_{j}\right| \log \left|z_{i}-z_{j}\right|+\frac{\alpha^{\prime}}{2} \sum_{m \neq i}\left(q k_{m}\right) \log \left|z_{i}-z_{j}\right| \log \left|z_{i}-z_{m}\right|\right]\right\} \\
& +\epsilon_{q}^{B \mu \nu} \sum_{i \neq j \neq m}^{n} k_{i \mu} k_{j \nu}\left(\frac{\alpha^{\prime}}{2}\right)^{2}\left(q k_{m}\right)\left[\operatorname{Li}_{2}\left(\frac{\bar{z}_{i}-\bar{z}_{m}}{\bar{z}_{i}-\bar{z}_{j}}\right)-\operatorname{Li}_{2}\left(\frac{z_{i}-z_{m}}{z_{i}-z_{j}}\right)\right. \\
& \left.+\log \frac{\left|z_{i}-z_{j}\right|}{\left|z_{i}-z_{m}\right|} \log \left(\frac{z_{m}-z_{j}}{\bar{z}_{m}-\bar{z}_{j}} \frac{\bar{z}_{i}-\bar{z}_{j}}{z_{i}-z_{j}}\right)\right]+\mathcal{O}\left(q^{2}\right),
\end{aligned}
$$

where

$$
\epsilon_{q}^{S \mu \nu}=\frac{\epsilon_{q}^{\mu} \bar{\epsilon}_{q}^{\nu}+\epsilon_{q}^{\nu} \bar{\epsilon}_{q}^{\mu}}{2}, \epsilon_{q}^{B \mu \nu}=\frac{\epsilon_{q}^{\mu} \bar{\epsilon}_{q}^{\nu}-\epsilon_{q}^{\nu} \bar{\epsilon}_{q}^{\mu}}{2} .
$$

For the next terms, only the parts up to order $q^{0}$ were derived previously. For completeness we express the full result, together with the new terms of order $q$ :

$$
\begin{aligned}
S_{1}^{(1)}= & -\epsilon_{q \mu} \bar{\epsilon}_{q \nu} \sqrt{\frac{\alpha^{\prime}}{2}} \sum_{i \neq j}\left[\frac { \theta _ { i } \epsilon _ { i } q } { z _ { i } - z _ { j } } \left(\frac{k_{j}^{\mu} k_{i}^{\nu}}{q k_{i}}-\frac{k_{j}^{\mu} k_{j}^{\nu}}{q k_{j}}+\alpha^{\prime}\left(k_{j}^{\mu} k_{i}^{\nu}-k_{j}^{\mu} k_{j}^{\nu}\right) \log \left|z_{i}-z_{j}\right|\right.\right. \\
& \left.+\frac{\alpha^{\prime}}{2} k_{i}^{\mu} k_{i}^{\nu} \frac{q k_{j}}{q k_{i}}-\frac{\alpha^{\prime}}{2} k_{i}^{\mu} k_{j}^{\nu}+\alpha^{\prime} k_{j}^{\mu} k_{i}^{\nu} \sum_{l \neq i} \frac{q k_{l}}{q k_{i}} \log \left|z_{i}-z_{l}\right|-\alpha^{\prime} k_{j}^{\mu} k_{j}^{\nu} \sum_{l \neq j} \frac{q k_{l}}{q k_{j}} \log \left|z_{j}-z_{l}\right|\right) \\
& \left.+\alpha^{\prime} k_{i}^{\mu} k_{j}^{\nu} \sum_{l \neq i j} \theta_{l} \epsilon_{l} q \frac{\log \left|z_{j}-z_{l}\right|-\log \left|z_{i}-z_{j}\right|}{z_{i}-z_{l}}\right]+ \text { c.c. },
\end{aligned}
$$




$$
\begin{aligned}
& S_{1}^{(2)}=\epsilon_{q \mu} \bar{\epsilon}_{q \nu} \frac{\alpha^{\prime}}{2} \sum_{i \neq j}^{n}\left[\frac{k_{j}^{\mu} k_{i}^{\nu}-k_{i}^{\mu} k_{i}^{\nu}}{q k_{i}} \frac{\left(\theta_{i} \epsilon_{i} q\right)\left(\theta_{j} \epsilon_{j} q\right)}{\left(z_{i}-z_{j}\right)^{2}}+\frac{k_{i}^{\mu} k_{j}^{\nu}}{2} \sum_{l \neq i, j}^{n} \frac{\left(\theta_{l} \epsilon_{l} q\right)\left(\bar{\theta}_{l} \bar{\epsilon}_{l} q\right)}{q k_{l}\left(z_{i}-z_{l}\right)\left(\bar{z}_{j}-\bar{z}_{l}\right)}\right. \\
& +\left(k_{i}^{\mu} k_{i}^{\nu}\left(\bar{\theta}_{j} \bar{\epsilon}_{j} q\right)+k_{i}^{\mu} k_{j}^{\nu}\left(\bar{\theta}_{i} \bar{\epsilon}_{i} q\right)\right) \frac{\theta_{j} \epsilon_{j} q}{2\left|z_{i}-z_{j}\right|^{2}}\left(\frac{1}{q k_{i}}+\frac{1}{q k_{j}}\right) \\
& \left.+\frac{\frac{k_{i}^{\mu} k_{i}^{\nu}}{2}\left(\theta_{j} \epsilon_{j} q\right)+k_{j}^{\mu} k_{i}^{\nu}\left(\theta_{i} \epsilon_{i} q\right)}{q k_{i}\left(z_{i}-z_{j}\right)} \sum_{l \neq i, j}\left(\frac{\theta_{l} \epsilon_{l} q}{\left(z_{i}-z_{l}\right)}+\frac{\bar{\theta}_{l} \bar{\epsilon}_{l} q}{\left(\bar{z}_{i}-\bar{z}_{l}\right)}\right)\right]+ \text { c.c. } \\
& S_{2}^{(0)}=\epsilon_{q \mu} \bar{\epsilon}_{q \nu} \frac{\alpha^{\prime}}{2} \sum_{i \neq j}^{n} \frac{\left(\theta_{i} \epsilon_{i}^{\mu}\right)}{\left|z_{i}-z_{j}\right|^{2}}\left\{\left(\bar{\theta}_{i} \bar{\epsilon}_{i}^{\nu}\right) q k_{j}\left(1+\frac{1}{2} \sum_{l \neq i} \frac{q k_{l}}{q k_{i}}\left[\frac{\bar{z}_{i}-\bar{z}_{j}}{\bar{z}_{i}-\bar{z}_{l}}+\frac{z_{i}-z_{j}}{z_{i}-z_{l}}\right]\right)\right. \\
& \left.-\left(\bar{\theta}_{j} \bar{\epsilon}_{j}^{\nu}\right) \sum_{l \neq i, j} q k_{l} \frac{\left(\bar{z}_{i}-\bar{z}_{l}\right)\left(z_{j}-z_{l}\right)}{\left(z_{i}-z_{l}\right)\left(\bar{z}_{j}-\bar{z}_{l}\right)}\right\} \text {, } \\
& S_{2}^{(1)}=\epsilon_{q \mu} \bar{\epsilon}_{q \nu} \sqrt{\frac{\alpha^{\prime}}{2}} \sum_{i \neq j}^{n}\left[\frac{\left(\left(\theta_{i} \epsilon_{i}^{\mu}\right)\left(\theta_{j} \epsilon_{j} q\right)-\left(\theta_{j} \epsilon_{j}^{\mu}\right)\left(\theta_{i} \epsilon_{i} q\right)\right)\left(\bar{\theta}_{i} \bar{\epsilon}_{i}^{\nu}\right)}{\left|z_{i}-z_{j}\right|^{2}\left(z_{i}-z_{j}\right)}\left(1+\sum_{l \neq i} \frac{q k_{l}}{q k_{i}} \frac{\bar{z}_{i}-\bar{z}_{j}}{\bar{z}_{i}-\bar{z}_{l}}\right)\right. \\
& \left.+\frac{\left(\theta_{i} \epsilon_{i}^{\mu}\right)\left(\bar{\theta}_{j} \bar{\epsilon}_{j}^{\nu}\right)}{\bar{z}_{i}-\bar{z}_{j}} \sum_{l \neq i, j}^{n} \frac{\left(\theta_{l} \epsilon_{l} q\right)\left(\bar{z}_{i}-\bar{z}_{l}\right)}{\left(z_{i}-z_{l}\right)^{2}\left(\bar{z}_{j}-\bar{z}_{l}\right)}\right]+ \text { c.c. }, \\
& S_{2}^{(2)}=\epsilon_{q \mu} \bar{\epsilon}_{q \nu} \sum_{i \neq j} \frac{\left(\theta_{i} \epsilon_{i}^{\mu}\right)\left(\theta_{j} \epsilon_{j} q\right)}{2\left(z_{i}-z_{j}\right)^{2}}\left[\frac { 1 } { q k _ { i } } \sum _ { l \neq i } \left(\frac{\left(\bar{\theta}_{i} \bar{\epsilon}_{i}^{\nu}\right)\left(\bar{\theta}_{l} \bar{\epsilon}_{l} q\right)-\left(\bar{\theta}_{l} \bar{\epsilon}_{l}^{\nu}\right)\left(\bar{\theta}_{i} \bar{\epsilon}_{i} q\right)}{\left(\bar{z}_{i}-\bar{z}_{l}\right)^{2}}\right.\right. \\
& \left.\left.+\frac{1}{q k_{j}} \sum_{l \neq j} \frac{\left(\bar{\theta}_{l} \bar{\epsilon}_{l}^{\nu}\right)\left(\bar{\theta}_{j} \bar{\epsilon}_{j} q\right)-\left(\bar{\theta}_{j} \bar{\epsilon}_{j}^{\nu}\right)\left(\bar{\theta}_{l} \bar{\epsilon}_{l} q\right)}{\left(\bar{z}_{l}-\bar{z}_{j}\right)^{2}}\right)\right]+ \text { c.c. },
\end{aligned}
$$

$$
\begin{aligned}
S_{3}^{(0)}= & \epsilon_{q \mu} \bar{\epsilon}_{q \nu} \sqrt{\frac{\alpha^{\prime}}{2}} \sum_{i \neq j}^{n} \alpha^{\prime} q_{\rho}\left[\frac{k_{i}^{\nu} k_{j}^{\rho}-k_{j}^{\nu} k_{i}^{\rho}}{z_{i}-z_{j}}\left(\theta_{i} \epsilon_{i}^{\mu}\right)\left(\frac{1}{\alpha^{\prime} q k_{i}}+\frac{1}{2}\right)\right. \\
& \left.+\sum_{l \neq i} \frac{k_{i}^{\nu} k_{l}^{\rho}-k_{l}^{\nu} k_{i}^{\rho}}{z_{i}-z_{j}}\left(\left(\theta_{j} \epsilon_{j}^{\mu}\right)+\left(\theta_{i} \epsilon_{i}^{\mu}\right) \frac{q k_{j}}{q k_{i}}\right) \log \left|z_{i}-z_{l}\right|\right]+ \text { c.c. },
\end{aligned}
$$$$
S_{3}^{(1)}=\epsilon_{q \mu} \bar{\epsilon}_{q \nu} \sum_{i \neq j}^{n}\left(\theta_{i} \epsilon_{i}^{\mu}\right)\left\{\frac { ( \theta _ { j } \epsilon _ { j } q ) } { ( z _ { i } - z _ { j } ) ^ { 2 } } \left[\frac{k_{i}^{\nu}}{k_{i} q}-\frac{k_{j}^{\nu}}{k_{j} q}-\alpha^{\prime} q_{\rho} \sum_{l \neq j} \frac{k_{j}^{\nu} k_{l}^{\rho}-k_{l}^{\nu} k_{j}^{\rho}}{q k_{j}} \log \left|z_{j}-z_{l}\right|\right.\right.
$$$$
\left.-\alpha^{\prime} q_{\rho} \sum_{l \neq i} \frac{k_{i}^{\nu} k_{l}^{\rho}-k_{l}^{\nu} k_{i}^{\rho}}{q k_{i}}\left(\frac{1}{2} \frac{z_{i}-z_{j}}{z_{i}-z_{l}}-\log \left|z_{i}-z_{l}\right|\right)\right]-\frac{\alpha^{\prime}}{2} \sum_{l \neq i, j} \frac{k_{j}^{\nu} \bar{\theta}_{l} \bar{\epsilon}_{l} q+k_{l}^{\nu} \bar{\theta}_{j} \bar{\epsilon}_{j} q}{\left(z_{i}-z_{l}\right)\left(\bar{z}_{j}-\bar{z}_{l}\right)}
$$$$
\left.-\frac{\alpha^{\prime}}{2} \frac{k_{i}^{\nu} \bar{\theta}_{j} \bar{\epsilon}_{j} q+k_{j}^{\nu} \bar{\theta}_{i} \bar{\epsilon}_{i} q}{\left|z_{i}-z_{j}\right|^{2}}\left(1+\sum_{l \neq i} \frac{q k_{l}}{q k_{i}} \frac{z_{i}-z_{j}}{z_{i}-z_{l}}\right)\right\}+ \text { c.c. }
$$ 


$$
\begin{aligned}
S_{3}^{(2)}= & \epsilon_{q \mu} \bar{\epsilon}_{q \nu} \sqrt{\frac{\alpha^{\prime}}{2}} \sum_{i \neq j}^{n}\left[\sum_{l \neq i, j}^{n} \frac{\left(\theta_{i} \epsilon_{i}^{\mu}\right)\left(\theta_{j} \epsilon_{j} q\right)\left(\theta_{l} \epsilon_{l} q\right)}{\left(z_{j}-z_{l}\right)\left(z_{i}-z_{j}\right)^{2}}\left(\frac{k_{j}^{\nu}}{q k_{j}}-\frac{k_{i}^{\nu}}{q k_{i}}\right)\right. \\
& \left.+\frac{\left(\theta_{j} \epsilon_{j}^{\mu}\right)\left(\theta_{i} \epsilon_{i} q\right)-\left(\theta_{i} \epsilon_{i}^{\mu}\right)\left(\theta_{j} \epsilon_{j} q\right)}{q k_{i}} \sum_{l \neq i} \frac{\left(k_{i}^{\nu} \bar{\theta}_{l} \bar{\epsilon}_{l} q+k_{l}^{\nu} \bar{\theta}_{i} \bar{\epsilon}_{i} q\right)}{\left(z_{i}-z_{j}\right)^{2}\left(\bar{z}_{i}-\bar{z}_{l}\right)}\right]+ \text { c.c. }
\end{aligned}
$$

As a nontrivial consistency check, it is possible to show that the full expression $S_{1}+S_{2}+S_{3}$ obeys gauge invariance, meaning that it vanishes identically by the replacement $\epsilon_{q \mu} \rightarrow q_{\mu}$ and $\bar{\epsilon}_{q \nu} \rightarrow q_{\nu}$. Actually, the identity is stronger, since the full expression vanishes by replacing only $\epsilon_{q \mu} \rightarrow q_{\mu}$ or $\bar{\epsilon}_{q \nu} \rightarrow q_{\nu}$, which can be explicitly checked from the above expression. In other words,

$$
q_{\mu} M_{n+1}^{\mu \nu}=q_{\nu} M_{n+1}^{\mu \nu}=0,
$$

where $M_{n+1}^{\mu \nu}$ is the stripped soft amplitude with respect to the polarization of the soft particle.

We want to find a gauge invariant operator that, when acting on $M_{n}$ reproduces the above results, i.e.

$$
M_{n+1}\left(q ; k_{i}\right)=M_{n}\left(k_{i}\right) * S\left(q, k_{i}\right)=\kappa_{D}\left(\hat{S}_{q}^{(-1)}+\hat{S}_{q}^{(0)}+\hat{S}_{q}^{(1)}\right) M_{n}\left(k_{i}\right)+\mathcal{O}\left(q^{2}\right),
$$

where the superscript of each $\hat{S}_{q}^{(m)}$ indicates the order $m$ in $q$ of each term. In ref. [78] we showed that the leading and subleading terms, symmetric in the polarization indices $\mu, \nu$, are generated by exactly the same soft-operators that one can infer using just gaugeinvariance of the amplitude, which read:

$$
\hat{S}_{q}^{(-1)}=\epsilon_{\mu \nu}^{S} \sum_{i=1}^{n} \frac{k_{i}^{\mu} k_{i}^{\nu}}{k_{i} \cdot q}, \quad \hat{S}_{q}^{(0)}=\epsilon_{\mu \nu}^{S}\left(-\frac{i q_{\rho}}{2}\right) \sum_{i=1}^{n} \frac{k_{i}^{\mu} J_{i}^{\nu \rho}+k_{i}^{\nu} J_{i}^{\mu \rho}}{k_{i} \cdot q},
$$

where

$$
\begin{array}{rlrl}
J_{i}^{\mu \nu} & =L_{i}^{\mu \nu}+\mathcal{S}_{i}^{\mu \nu}, & \mathcal{S}_{i}^{\mu \nu} & =S_{i}^{\mu \nu}+\bar{S}_{i}^{\mu \nu}, \\
L_{i}^{\mu \nu} & =i\left(k_{i}^{\mu} \frac{\partial}{\partial k_{i \nu}}-k_{i}^{\nu} \frac{\partial}{\partial k_{i \mu}}\right), \quad S_{i}^{\mu \nu}=i\left(\epsilon_{i}^{\mu} \frac{\partial}{\partial \epsilon_{i \nu}}-\epsilon_{i}^{\nu} \frac{\partial}{\partial \epsilon_{i \mu}}\right), \quad \bar{S}_{i}^{\mu \nu}=i\left(\bar{\epsilon}_{i}^{\mu} \frac{\partial}{\partial \bar{\epsilon}_{i \nu}}-\bar{\epsilon}_{i}^{\nu} \frac{\partial}{\partial \bar{\epsilon}_{i \mu}}\right) .
\end{array}
$$

The new result here is that the subsubleading terms, symmetric in the polarization indices $\mu, \nu$, are uniquely generated by the following soft operator, which can be explicitly checked:

$$
\begin{aligned}
S_{q}^{(1)}= & -\frac{\epsilon_{\mu \nu}^{S}}{2} \sum_{i=1}^{n}\left[\frac{q_{\rho} J_{i}^{\mu \rho} q_{\sigma} J_{i}^{\nu \sigma}}{k_{i} \cdot q}+\left(\frac{k_{i}^{\mu} q^{\nu}}{k_{i} \cdot q} q^{\sigma}+q^{\mu} \eta^{\nu \sigma}-\eta^{\mu \nu} q^{\sigma}\right) \frac{\partial}{\partial k_{i}^{\sigma}}\right. \\
& -\left(\frac{q_{\rho} q_{\sigma} \eta_{\mu \nu}-q_{\sigma} q_{\nu} \eta_{\rho \mu}-q_{\rho} q_{\mu} \eta_{\sigma \nu}}{k_{i} \cdot q}\right)\left(\epsilon_{i}^{\rho} \frac{\partial}{\partial \epsilon_{i \sigma}}+\bar{\epsilon}_{i}^{\rho} \frac{\partial}{\partial \bar{\epsilon}_{i \sigma}}\right) \\
& \left.-\alpha^{\prime}\left(q_{\sigma} k_{i \nu} \eta_{\rho \mu}+q_{\rho} k_{i \mu} \eta_{\sigma \nu}-\eta_{\rho \mu} \eta_{\sigma \nu}\left(k_{i} \cdot q\right)-q_{\rho} q_{\sigma} \frac{k_{i \mu} k_{i \nu}}{k_{i} \cdot q}\right)\left(\epsilon_{i}^{\rho} \frac{\partial}{\partial \epsilon_{i \sigma}}+\bar{\epsilon}_{i}^{\rho} \frac{\partial}{\partial \bar{\epsilon}_{i \sigma}}\right)\right] .
\end{aligned}
$$


It is thus useful to also define:

$$
\Pi_{i}^{\rho \sigma}=\epsilon_{i}^{\rho} \frac{\partial}{\partial \epsilon_{i \sigma}}+\bar{\epsilon}_{i}^{\rho} \frac{\partial}{\partial \bar{\epsilon}_{i \sigma}} .
$$

Notice that only the symmetric combination $\Pi_{i}^{\{\rho, \sigma\}}=\frac{\Pi_{i}^{\rho \sigma}+\Pi_{i}^{\sigma \rho}}{2}$ survives the contractions in eq. (2.24), since the contraction of $\mu$ and $\nu$ is symmetric.

The terms in the first two lines of eq. (2.24), which are finite in the field theory limit, exactly match the soft theorem derived in ref. [24] using just on-shell gauge invariance of tree-level gravity amplitudes. The terms in the last line can thus be seen as the string corrections to the field theory soft theorem. Notice that each parenthesis is independently gauge invariant. Notice also that in the field theory limit, if the soft particle is a graviton, only the first term is nonzero, since $\epsilon_{\mu \nu}^{\text {graviton }}$ is traceless. The extra terms in the first line were found already in ref. [78] for the case, where the $n$ external states were tachyons.

In ref. [78], we also found a soft theorem for the antisymmetric part at the subleading order, corresponding to a soft Kalb-Ramond field. At this point, however, it is not clear how the antisymmetric part of our subsubleading explicit results could also be expressed as a soft theorem, since at this order dilogarithmic terms appear in eq. (2.13). We thus leave the analysis of the antisymmetric part for a possible future study.

In the next section we specify the subsubleading operator to the case of a soft dilaton and a soft graviton and we give a physical interpretation of the various terms that appear.

\section{Soft gravitons and dilatons}

Specifying our main result eq. (2.24) to the cases where the soft particle is either a graviton or a dilaton, we may first simplify the general expression by imposing the transversality condition $\epsilon_{\mu \nu}^{S} q^{\mu}=\epsilon_{\mu \nu}^{S} q^{\nu}=0$, leading to:

$$
\begin{aligned}
S_{q}^{(1)}= & -\frac{\epsilon_{\mu \nu}^{S}}{2} \sum_{i=1}^{n}\left[\frac{q_{\rho} J_{i}^{\mu \rho} q_{\sigma} J_{i}^{\nu \sigma}}{k_{i} \cdot q}-\frac{\eta^{\mu \nu} q_{\rho} q_{\sigma}}{k_{i} \cdot q}\left(k_{i}^{\rho} \frac{\partial}{\partial k_{i \sigma}}+\Pi_{i}^{\{\rho, \sigma\}}\right)\right. \\
& \left.-\alpha^{\prime}\left(q_{\sigma} k_{i \nu} \eta_{\rho \mu}+q_{\rho} k_{i \mu} \eta_{\sigma \nu}-\eta_{\rho \mu} \eta_{\sigma \nu}\left(k_{i} \cdot q\right)-q_{\rho} q_{\sigma} \frac{k_{i \mu} k_{i \nu}}{k_{i} \cdot q}\right) \Pi_{i}^{\{\rho, \sigma\}}\right] .
\end{aligned}
$$

Considering the soft particle to be a graviton, tracelessness of its polarization gives:

$$
\begin{aligned}
S_{\text {graviton }, q}^{(1)}= & -\frac{\epsilon_{\mu \nu}^{\text {graviton }}}{2} \sum_{i=1}^{n}\left[\frac{q_{\rho} J_{i}^{\mu \rho} q_{\sigma} J_{i}^{\nu \sigma}}{k_{i} \cdot q}\right. \\
& \left.-\alpha^{\prime}\left(q_{\sigma} k_{i \nu} \eta_{\rho \mu}+q_{\rho} k_{i \mu} \eta_{\sigma \nu}-\eta_{\rho \mu} \eta_{\sigma \nu}\left(k_{i} \cdot q\right)-q_{\rho} q_{\sigma} \frac{k_{i \mu} k_{i \nu}}{k_{i} \cdot q}\right) \Pi_{i}^{\{\rho, \sigma\}}\right]
\end{aligned}
$$

The first term reproduces the subsubleading soft theorem of gravitons. The second line are the string corrections to the field theory result. We can reduce the derivatives with respect to $\epsilon_{i}$ and $\bar{\epsilon}_{i}$ in $\Pi_{i}$ by acting on the $n$-point amplitude with the polarization vectors stripped off, i.e.

$$
M_{n}\left(k_{i}, \epsilon_{i}, \bar{\epsilon}_{i}\right)=\epsilon_{1}^{\mu_{1}} \bar{\epsilon}_{1}^{\nu_{1}} \cdots \epsilon_{n}^{\mu_{n}} \bar{\epsilon}_{n}^{\nu_{n}} M_{n,\left(\mu_{1}, \nu_{1}\right), \ldots,\left(\mu_{n}, \nu_{n}\right)}\left(k_{i}\right)
$$


Then we can express:

$$
\begin{aligned}
\left(\epsilon_{i}^{\rho} \frac{\partial}{\partial \epsilon_{i \sigma}}+\bar{\epsilon}_{i}^{\rho} \frac{\partial}{\partial \bar{\epsilon}_{i \sigma}}\right) M_{n} & =\left(\eta^{\sigma \mu_{i}} \epsilon_{i}^{\rho} \bar{\epsilon}_{i}^{\nu_{i}}+\eta^{\sigma \nu_{i}} \epsilon_{i}^{\mu_{i}} \bar{\epsilon}_{i}^{\rho}\right) M_{n,\left(\mu_{i} \nu_{i}\right)} \\
& =2 \eta^{\sigma \mu_{i}}\left(\epsilon_{i}^{\left\{\rho, \bar{\epsilon}_{i}^{\left.\nu_{i}\right\}}\right.} M_{n,\left\{\mu_{i}, \nu_{i}\right\}}+\epsilon_{i}^{\left[\rho, \bar{\epsilon}_{i}^{\left.\nu_{i}\right]}\right.} M_{n,\left[\mu_{i}, \nu_{i}\right]}\right)
\end{aligned}
$$

where in the second line we decomposed $M_{n}$ into its symmetric and antisymmetric parts, as in eq. (2.14), showing that string corrections can exist for external states $i$ being polarized both symmetrically (gravitons and dilatons) and antisymmetrically (Kalb-Ramond). We will comment further on these new string-theory terms in the next section.

Projecting instead the soft leg onto the dilaton, using $\epsilon_{\mu \nu}^{d}=\left(\eta_{\mu \nu}-q_{\mu} \bar{q}_{\nu}-q_{\nu} \bar{q}_{\mu}\right) / \sqrt{D-2}$, with $q \cdot \bar{q}=1$ and $\bar{q}^{2}=q^{2}=0$, we get:

$$
\begin{aligned}
S_{\text {dilaton }, q}^{(1)}=\frac{1}{2 \sqrt{D-2}} \sum_{i=1}^{n} & {\left[q^{\rho} \hat{K}_{i \rho}+\frac{q^{\rho} q^{\sigma}}{k_{i} q}\left(\mathcal{S}_{i, \rho \mu} \eta^{\mu \nu} \mathcal{S}_{i \nu \sigma}+D \Pi_{i,\{\rho, \sigma\}}\right)\right.} \\
& \left.-\alpha^{\prime}\left(k_{i} \cdot q\right)\left(\epsilon_{i} \cdot \frac{\partial}{\partial \epsilon_{i}}+\bar{\epsilon}_{i} \cdot \frac{\partial}{\partial \bar{\epsilon}_{i}}\right)\right],
\end{aligned}
$$

where both $k_{i} \cdot \epsilon_{i}=k_{i} \cdot \bar{\epsilon}_{i}=0$ and gauge invariance, i.e. $k_{i} \cdot \frac{\partial}{\partial \epsilon_{i}} M_{n}=k_{i}^{\mu} M_{n, \mu}=0$ and $k_{i} \cdot \frac{\partial}{\partial \bar{\epsilon}_{i}} M_{n}=k_{i}^{\nu} M_{n, \nu}=0$, were used, and where we introduced the operator:

$$
\hat{K}_{i \mu}=2\left[\frac{1}{2} k_{i \mu} \frac{\partial^{2}}{\partial k_{i \nu} \partial k_{i}^{\nu}}-k_{i}^{\rho} \frac{\partial^{2}}{\partial k_{i}^{\mu} \partial k_{i}^{\rho}}+i \mathcal{S}_{i, \rho \mu} \frac{\partial}{\partial k_{i}^{\rho}}\right]
$$

Remarkably, this is exactly the generator of special conformal transformations acting on momentum space. The string correction for the dilaton vanishes due to momentum conservation, since the operator $\epsilon_{i} \cdot \frac{\partial}{\partial \epsilon_{i}}$ leaves $M_{n}$ invariant, yielding (correspondingly for the barred term)

$$
\frac{\alpha^{\prime}}{2} \sum_{i=1}^{n}\left(k_{i} \cdot q\right) \epsilon_{i} \cdot \frac{\partial}{\partial \epsilon_{i}} M_{n}=\frac{\alpha^{\prime}}{2} \sum_{i=1}^{n}\left(k_{i} \cdot q\right) M_{n}=-\frac{\alpha^{\prime}}{2} q^{2} M_{n}=0 .
$$

In conclusion, we find that the subsubleading dilaton soft operator equals the field theory counterpart and reads:

$$
S_{\text {dilaton }, q}^{(1)}=\frac{1}{2 \sqrt{D-2}} \sum_{i=1}^{n}\left[q^{\rho} \hat{K}_{i \rho}+\frac{q^{\rho} q^{\sigma}}{k_{i} q}\left(\mathcal{S}_{i, \rho \mu} \eta^{\mu \nu} \mathcal{S}_{i \nu \sigma}+D \Pi_{i,\{\rho, \sigma\}}\right)\right] .
$$

The subsubleading dilaton soft theorem contains a finite piece, which can be fully expressed by the generator of a special conformal transformation and a singular piece only dependent on polarization derivatives.

We may use the polarization-stripped form of $M_{n}$ in eq. (3.3) to understand the singular terms, which then after some simplification read (suppressing for brevity the factor 
$1 / \sqrt{D-2})$ :

$$
\begin{aligned}
& \sum_{i=1}^{n} \frac{q^{\rho} q^{\sigma}}{2 k_{i} q}\left(\mathcal{S}_{i, \rho \mu} \eta^{\mu \nu} \mathcal{S}_{i \nu \sigma}+D \Pi_{i,\{\rho, \sigma\}}\right) M_{n} \\
& =\sum_{i=1}^{n} \frac{1}{k_{i} q}\left(q_{\mu_{i}} q_{\nu_{i}}\left(\epsilon_{i} \cdot \bar{\epsilon}_{i}\right)+\eta_{\mu_{i} \nu_{i}}\left(q \cdot \epsilon_{i}\right)\left(q \cdot \bar{\epsilon}_{i}\right)\right. \\
& \left.\quad+\left(q \cdot \epsilon_{i}\right)\left(q_{\mu_{i}} \bar{\epsilon}_{\nu_{i}}-q_{\nu_{i}} \bar{\epsilon}_{\mu_{i}}\right)-\left(q \cdot \bar{\epsilon}_{i}\right)\left(q_{\mu_{i}} \epsilon_{\nu_{i}}-q_{\nu_{i}} \epsilon_{\mu_{i}}\right)\right) M_{n}^{\left(\mu_{i}, \nu_{i}\right)}
\end{aligned}
$$

The expression evidently separates into a symmetric and an antisymmetric part, which we can express using $M_{n}^{\mu_{i} \nu_{i}}=M_{n}^{\left\{\mu_{i}, \nu_{i}\right\}}+M_{n}^{\left[\mu_{i}, \nu_{i}\right]}$, reducing the previous expression to:

$$
\begin{aligned}
& \sum_{i=1}^{n}\left(\frac{q_{\mu_{i}} q_{\nu_{i}} \eta_{\alpha \beta}+q_{\alpha} q_{\beta} \eta_{\mu_{i} \nu_{i}}}{k_{i} \cdot q}\right) \epsilon_{i}^{\alpha} \bar{\epsilon}_{i}^{\beta} M_{n}^{\left\{\mu_{i}, \nu_{i}\right\}}+\sum_{i=1}^{n} 2\left(\frac{q_{\alpha} q_{\mu_{i}} \eta_{\beta \nu_{i}}+q_{\beta} q_{\nu_{i}} \eta_{\alpha \mu_{i}}}{k_{i} \cdot q}\right) \epsilon_{i}^{\alpha} \epsilon_{i}^{\beta} M_{n}^{\left[\mu_{i}, \nu_{i}\right]} \\
& =\sum_{i=1}^{n}\left(\frac{q_{\mu_{i}} q_{\nu_{i}} \eta_{\alpha \beta}+q_{\alpha} q_{\beta} \eta_{\mu_{i} \nu_{i}}}{k_{i} \cdot q}\right) \epsilon_{i}^{S \alpha, \beta} M_{n}^{\left\{\mu_{i}, \nu_{i}\right\}}+\sum_{i=1}^{n} 4 \frac{q_{\alpha} q_{\mu_{i}} \eta_{\beta \nu_{i}}}{k_{i} \cdot q} \epsilon_{i}^{B \alpha, \beta} M_{n}^{\left[\mu_{i}, \nu_{i}\right]}
\end{aligned}
$$

where in the second line we also decomposed the polarization vectors as in eq. (2.14). The form of these terms suggest that they are coming from factorizing exchange diagrams, where the soft dilaton is attached to an external leg through a three-point vertex, with the indices $\alpha, \beta$ being the polarization indices of the internal state. In the field theory limit there are only two types of such vertices, one involving two dilatons derivatively coupled to a graviton and two Kalb-Ramond fields derivatively coupled to one dilaton, giving rise to the three types of factorizing diagrams shown in figure 1. Indeed, if we project the external leg $i$ on each of the three massless states, the expression above reduces in each case to one nonzero term:

$$
\begin{array}{ll}
\text { For } \epsilon_{i}^{\alpha \beta}=\epsilon_{g}^{\alpha \beta}: & \frac{q_{\alpha} q_{\beta}}{k_{i} \cdot q} \eta_{\mu_{i} \nu_{i}} \epsilon_{g}^{\alpha, \beta} M_{n}^{\left\{\mu_{i}, \nu_{i}\right\}} . \\
\text { For } \epsilon_{i}^{\alpha \beta}=\epsilon_{d}^{\alpha \beta}: & \frac{q_{\mu_{i}} q_{\nu_{i}}}{k_{i} \cdot q} \eta_{\alpha \beta} \epsilon_{d}^{\alpha, \beta} M_{n}^{\left\{\mu_{i}, \nu_{i}\right\}} . \\
\text { For } \epsilon_{i}^{\alpha \beta}=\epsilon_{B}^{\alpha \beta}: & 4 \frac{q_{\alpha} q_{\mu_{i}}}{k_{i} \cdot q} \eta_{\beta \nu_{i}} \epsilon_{B}^{\alpha, \beta} M_{n}^{\left[\mu_{i}, \nu_{i}\right]} .
\end{array}
$$

It is worth noticing from figure 1 that in the cases where the $i$ th particle is either a dilaton or a graviton, there are contributions to the dilaton soft theorem, where the $i$ th particle in the lower point amplitude $M_{n}$ is changed to respectively a graviton or a dilaton. This means that the subsubleading term of the soft dilaton amplitude separates into two factorized terms when the $i$ th state is a dilaton or a graviton. Specifically, for either of the three possible cases we have:

$$
\begin{aligned}
M_{n+1}\left(\phi(q) ; \phi\left(k_{i}\right), \ldots\right) & \sim\left(\hat{S}^{(0)}+q^{\mu} \hat{S}_{\mu}^{(1)}\right) M_{n}\left(\phi\left(k_{i}\right), \ldots\right)+q^{\mu} \hat{S}_{\mu}^{\phi g} M_{n}\left(g_{\alpha \beta}\left(k_{i}\right), \ldots\right)+\mathcal{O}\left(q^{2}\right), \\
M_{n+1}\left(\phi(q) ; g_{\mu_{i} \nu_{i}}\left(k_{i}\right), \ldots\right) & \sim\left(\hat{S}^{(0)}+q^{\mu} \hat{S}_{\mu}^{(1)}\right) M_{n}\left(g_{\mu_{i} \nu_{i}}\left(k_{i}\right), \ldots\right)+q^{\mu} \hat{S}_{\mu}^{g \phi} M_{n}\left(\phi\left(k_{i}\right), \ldots\right)+\mathcal{O}\left(q^{2}\right), \\
M_{n+1}\left(\phi(q) ; B_{\mu_{i} \nu_{i}}\left(k_{i}\right), \ldots\right) & \sim\left(\hat{S}^{(0)}+q^{\mu} \hat{S}_{\mu}^{(1)}\right) M_{n}\left(B_{\mu_{i} \nu_{i}}\left(k_{i}\right), \ldots\right)+\mathcal{O}\left(q^{2}\right),
\end{aligned}
$$



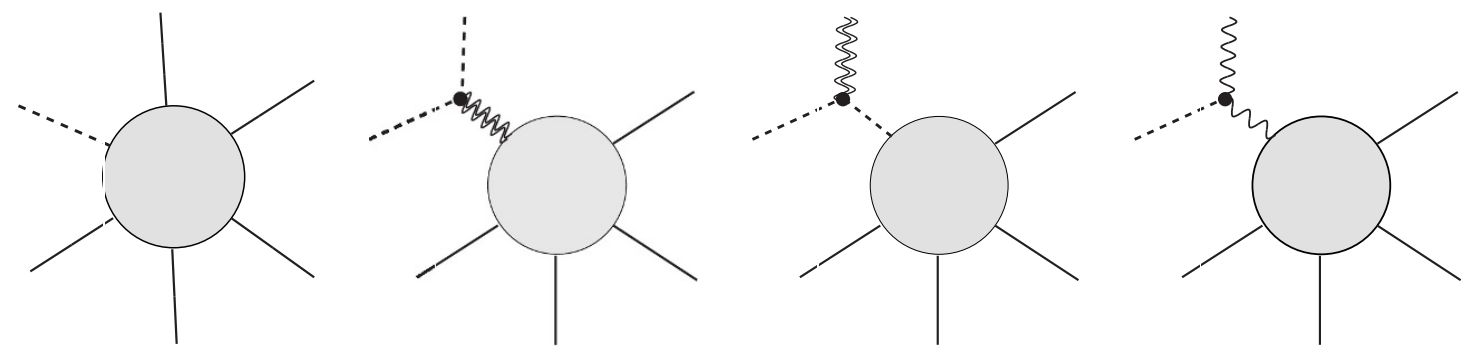

Figure 1. Soft dilaton (dashed line) scattering on other massless states (solid lines), and the only three types of exchange diagrams appearing in field theory, involving i) another external dilaton and an internal graviton (double-wavy line), ii) an external graviton and an internal dilaton, iii) an external and an internal Kalb-Ramond field (wavy line).

where $\phi$ denotes a dilaton, $g_{\alpha \beta}$ denotes a graviton, and $B_{\alpha \beta}$ denotes a Kalb-Ramond field. The non-standard factorizing behavior of the first two cases was also noticed in ref. [63] in specific examples.

In conclusion, in this section we have provided a physical interpretation of the subsubleading terms that survive in the field theory limit. In the next section we extend our analysis to the terms with string corrections. In particular, we show that they are completely determined from gauge invariance and from the string correction terms, appearing in the bosonic string, in the three-point amplitude involving massless particles.

\section{String corrections from gauge invariance}

In this section we derive the string corrections to the soft operator, found in the previous section from explicit calculations, by considering the string corrections to the three-point amplitude of the bosonic string involving three massless closed string and exploiting onshell gauge invariance of the amplitude. Let us consider the three-point amplitude of three massless bosonic strings with the given set of momenta and polarizations:

$$
\left(q, \epsilon_{q}^{\mu}, \bar{\epsilon}_{q}^{\nu}\right), \quad\left(k_{i}, \epsilon_{i}^{\mu_{i}}, \bar{\epsilon}_{i}^{\nu_{i}}\right), \quad\left(-k_{i}-q, \epsilon_{m}^{\alpha}, \bar{\epsilon}_{m}^{\beta}\right)
$$

The polarization stripped three-point on-shell amplitude then reads:

$$
\begin{aligned}
M_{3}^{\mu \nu ; \mu_{i} \nu_{i} ; \alpha \beta}= & 2 \kappa_{D}\left(\eta^{\mu \mu_{i}} q^{\alpha}-\eta^{\mu \alpha} q^{\mu_{i}}+\eta^{\mu_{i} \alpha} k_{i}^{\mu}-\frac{\alpha^{\prime}}{2} k_{i}^{\mu} q^{\mu_{i}} q^{\mu}\right) \\
& \times\left(\eta^{\nu \nu_{i}} q^{\beta}-\eta^{\nu \beta} q^{\nu_{i}}+\eta^{\nu_{i} \beta} k_{i}^{\nu}-\frac{\alpha^{\prime}}{2} k_{i}^{\nu} q^{\nu_{i}} q^{\nu}\right) .
\end{aligned}
$$

Contracting this expression with particular polarization tensors yields the explicit expression for particular three-point amplitudes of massless strings. For instance, considering the case where one of the states is a dilaton, and contracting with the polarization tensor used in eq. (3.5), we get the following nonzero three-point amplitudes involving one dilaton:

$$
\begin{aligned}
& M_{d d g}=2 \kappa_{D} \epsilon_{g}^{\alpha \beta} q_{\alpha} q_{\beta}, \quad M_{d B B}=\frac{2 \kappa_{D}}{\sqrt{D-2}} 4 \epsilon_{B}^{\mu_{i} \nu_{i}} \eta_{\mu_{i} \alpha} \epsilon_{B}^{\alpha \beta} q_{\nu_{i}} q_{\beta}, \\
& M_{d g g}=-\alpha^{\prime} \frac{2 \kappa_{D}}{\sqrt{D-2}} \epsilon_{g}^{\mu_{i} \nu_{i}} \epsilon_{g}^{\alpha \beta} q_{\mu_{i}} q_{\nu_{i}} q_{\alpha} q_{\beta} .
\end{aligned}
$$


Notice that the dilaton-graviton-graviton amplitude vanishes in the field theory limit. From these three-point amplitudes we can immediately write down the contributions from the factorizing exchange diagrams in figure 1 to the soft theorem, i.e.

$$
\begin{aligned}
& M_{n+1}^{\text {ex. }}\left(\phi(q), \phi\left(k_{i}\right), \ldots\right) \\
& \sim M_{d d g} \frac{1}{\left(k_{i}+q\right)^{2}} M_{n}\left(g_{\alpha \beta}\left(k_{i}+q\right), \ldots\right)=\kappa_{D} \frac{q_{\alpha} q_{\beta}}{k_{i} \cdot q} \epsilon_{g}^{\alpha \beta} M_{n}\left(g_{\alpha \beta}\left(k_{i}+q\right), \ldots\right) \\
& M_{n+1}^{\text {ex. }}\left(\phi(q), g_{\mu_{i} \nu_{i}}\left(k_{i}\right), \ldots\right) \\
& \sim M_{d d g} \frac{1}{\left(k_{i}+q\right)^{2}} M_{n}\left(\phi\left(k_{i}+q\right), \ldots\right)+M_{d g g} \frac{1}{\left(k_{i}+q\right)^{2}} M_{n}\left(g_{\alpha \beta}\left(k_{i}+q\right), \ldots\right) \\
& =\kappa_{D} \frac{q_{\mu_{i}} q_{\nu_{i}}}{k_{i} \cdot q} \epsilon_{g}^{\mu_{i} \nu_{i}} M_{n}\left(\phi\left(k_{i}+q\right), \ldots\right)-\frac{\alpha^{\prime} \kappa_{D}}{\sqrt{D-2}} \epsilon_{g}^{\mu_{i} \nu_{i}} \frac{q_{\mu_{i}} q_{\nu_{i}} q_{\alpha} q_{\beta}}{k_{i} \cdot q} \epsilon_{g}^{\alpha \beta} M_{n}\left(g_{\alpha \beta}\left(k_{i}+q\right), \ldots\right) \\
& M_{n+1}^{\text {ex. }}\left(\phi(q), B_{\mu_{i} \nu_{i}}\left(k_{i}\right), \ldots\right) \\
& \sim \frac{M_{d B B}}{\left(k_{i}+q\right)^{2}} M_{n}\left(B_{\alpha \beta}\left(k_{i}+q\right), \ldots\right)=\frac{\kappa_{D}}{\sqrt{D-2}} \epsilon_{B}^{\mu_{i} \nu_{i}} \frac{4 q_{\nu_{i}} q_{\beta} \eta_{\mu_{i} \alpha}}{k_{i} \cdot q} \epsilon_{B}^{\alpha \beta} M_{n}\left(B_{\alpha \beta}\left(k_{i}+q\right), \ldots\right) .
\end{aligned}
$$

These expressions match through order $\mathcal{O}(q)$ exactly the singular terms in the dilaton soft theorem, found in the previous section. Specifically, in eq. (3.11) (where a factor $\kappa_{D} / \sqrt{D-2}$ from eqs. (2.20) and (3.8) was suppressed) one should make the identifications:

$$
\begin{aligned}
M_{n}^{\left\{\mu_{i}, \nu_{i}\right\}} & \equiv \epsilon_{g}^{\mu_{i} \nu_{i}} M_{n}\left(g_{\alpha \beta}\left(k_{i}\right), \ldots\right)+\epsilon_{d}^{\mu_{i} \nu_{i}} M_{n}\left(\phi\left(k_{i}\right), \ldots\right), \\
M_{n}^{\left[\mu_{i}, \nu_{i}\right]} & \equiv \epsilon_{B}^{\mu_{i} \nu_{i}} M_{n}\left(B_{\alpha \beta}\left(k_{i}\right), \ldots\right) .
\end{aligned}
$$

Notice that the three-point amplitude proportional to $\alpha^{\prime}$, involving one dilaton and two gravitons, does not contribute to the dilaton soft theorem, since it is proportional to the fourth power in the soft momentum, and thus contributes at the order $q^{3}$.

Let us now consider the $\alpha^{\prime}$-correction terms to the graviton soft theorem appearing at subsubleading order in eq. (3.2). Among them there is a term with the propagator-pole $1 / k_{i} \cdot q$, which should come from a factorizing exchange diagram. Indeed, expanding the three-point amplitude in terms of $q$, with the soft-state now being a graviton, the leading string-correction to the three point amplitude reads:

$$
\begin{aligned}
\left.M_{3}^{\mu \nu, \alpha \beta}\right|_{\alpha^{\prime}} & \sim-\left(2 \kappa_{D}\right) \frac{\alpha^{\prime}}{2} k_{i}^{\mu} k_{i}^{\nu}\left(\left(\epsilon_{i} \cdot q\right) q^{\alpha} \bar{\epsilon}^{\beta}+\left(\bar{\epsilon}_{i} \cdot q\right) q^{\beta} \epsilon_{i}^{\alpha}\right)+\mathcal{O}\left(q^{3}\right) \\
& =-\left(2 \kappa_{D}\right) \frac{\alpha^{\prime}}{2} k_{i}^{\mu} k_{i}^{\nu} q_{\rho} q_{\sigma}\left(\eta^{\sigma \alpha} \epsilon_{i}^{\rho} \bar{\epsilon}_{i}^{\beta}+\eta^{\sigma \beta} \epsilon_{i}^{\alpha} \bar{\epsilon}_{i}^{\rho}\right)+\mathcal{O}\left(q^{3}\right)
\end{aligned}
$$

where we contracted with the polarization vectors of leg $i$. Comparing with eqs. (3.2) and (3.4), we see that the singular string correction in eq. (3.2) is exactly reproduced by the exchange diagram given by $\frac{M_{3}}{\left(k_{i}+q\right)^{2}} M_{n}$, with the singularity coming from the pole of the exchanged state. 


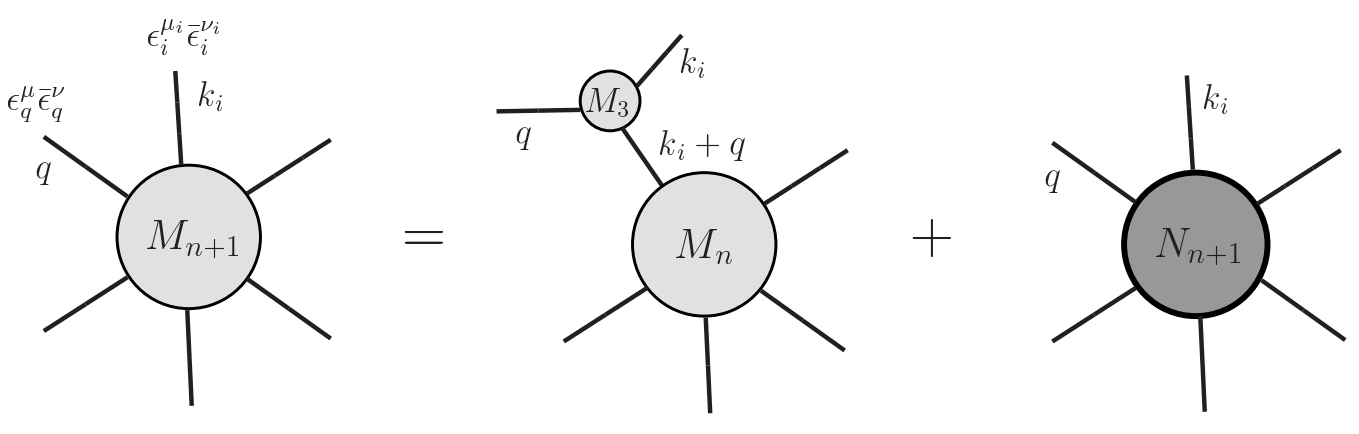

Figure 2. Decomposition of the $n+1$-point massless closed string amplitude, $M_{n+1}$, into a factorizing part involving the three-point amplitude $M_{3}$, where the soft state with momentum $q$ directly interacts with the $i$ th state, exchanging a third massless closed state with momentum $k_{i}+q$ with the $n$-point amplitude $M_{n}$, and the reminding part of the amplitude, $N_{n+1}$, which excludes factorization through the former channel.

We will now show that the remaining factorizing non-singular terms in eq. (3.1) all follow from on-shell gauge invariance of the amplitude.

By using the expression for the three-point amplitude in eq. (4.2), specialized to gravitons in the field theory limit $\alpha^{\prime} \rightarrow 0$, it has been shown in ref. [62] that by decomposing the $n+1$ point amplitude as in figure 2, and imposing gauge invariance, i.e. $q_{\mu} M_{n+1}^{\mu \nu}=q_{\nu} M_{n+1}^{\mu \nu}=0$, one exactly recovers the tree-level soft graviton theorem to subsubleading order. In the previous sections we have explicitly shown to subsubleading order and for a finite value of $\alpha^{\prime}$ (see also ref. [78]) that the same gauge invariance also applies to amplitudes where the external states can, as well as gravitons, be dilatons and KalbRamond states. Based on this, it was shown in ref. [24] that in the limit $\alpha^{\prime} \rightarrow 0$ the dilaton soft theorem to subsubleading order can be found also using just gauge invariance of the on-shell amplitude. (In ref. [24] only the cases where the other hard states are either dilatons or gravitons were considered, however, the extension to also include the antisymmetric Kalb-Ramond states trivially yields the expression found in eq. (3.8).) Now we extend this line of analysis to also involve the string corrections to the three-point amplitude.

We first decompose the $n+1$-point amplitude as in figure 2, reading:

$$
M_{n+1}^{\mu \nu}=\sum_{i=1}^{n} M_{3}^{\mu \nu}\left(q ; k_{i}\right) \frac{1}{\left(k_{i}+q\right)^{2}} M_{n}\left(k_{i}+q\right)+N^{\mu \nu}\left(q ; k_{i}\right),
$$

where dependence on all other $k_{j} \neq k_{i}$ is implicit. The indices $\mu \nu$ belong to the soft state with momentum $q$, and we assume them to be symmetric, i.e. $M_{n+1}^{\mu \nu}=M_{n+1}^{\nu \mu}$. We now use the explicit form of the three-point string amplitude, $M_{3}$, and focus here only on the $\alpha^{\prime}$-terms. We can read off these terms, when $\mu \nu$ is symmetric, from eq. (4.8), and as we noticed before, they can be rewritten as an operator by using eq. (3.4), when they are multiplied $M_{n}$. Thus we have:

$$
\left.M_{n+1}^{\mu \nu}\right|_{\alpha^{\prime}}=-\frac{\alpha^{\prime}}{2} \sum_{i=1}^{n} \frac{k_{i}^{\mu} k_{i}^{\nu}}{k_{i} \cdot q} q_{\rho} q_{\sigma} \Pi_{i}^{\{\rho, \sigma\}} M_{n}\left(k_{i}\right)+\left.N^{\mu \nu}\left(q ; k_{i}\right)\right|_{\alpha^{\prime}}+\mathcal{O}\left(q^{2}\right),
$$


Now, imposing on-shell gauge invariance of the amplitude, we first find from $q_{\mu} M_{n+1}^{\mu \nu}=0$, and Taylor expanding in $q$ :

$$
\begin{aligned}
\left.N^{\mu \nu}\left(q=0, k_{i}\right)\right|_{\alpha^{\prime}} & =0 \\
\left.\frac{1}{2}\left(\frac{\partial N^{\mu \nu}}{\partial q_{\rho}}+\frac{\partial N^{\rho \nu}}{\partial q_{\mu}}\right)\right|_{\alpha^{\prime}, q=0} & =\alpha^{\prime} \sum_{i=1}^{n} k_{i}^{\nu} \Pi_{i}^{\{\rho, \mu\}} M_{n}\left(k_{i}\right) .
\end{aligned}
$$

Inserting this back into the Taylor-expanded form of eq. (4.10), and imposing also $q_{\nu} M_{n+1}^{\mu \nu}=0$, we get:

$$
\left.\frac{1}{2}\left(\frac{\partial N^{\mu \nu}}{\partial q_{\rho}}-\frac{\partial N^{\rho \nu}}{\partial q_{\mu}}\right)\right|_{\alpha^{\prime}, q=0}=\frac{\alpha^{\prime}}{2} \sum_{i=1}^{n}\left(k_{i}^{\mu} \Pi_{i}^{\{\rho, \nu\}}-k_{i}^{\nu} \Pi_{i}^{\{\rho, \mu\}}-k_{i}^{\rho} \Pi_{i}^{\{\mu, \nu\}}\right) M_{n}\left(k_{i}\right) .
$$

Thus, in total we find:

$\left.M_{n+1}^{\mu \nu}\right|_{\alpha^{\prime}}=-\frac{\alpha^{\prime}}{2} q_{\rho} \sum_{i=1}^{n}\left(\frac{k_{i}^{\mu} k_{i}^{\nu}}{k_{i} \cdot q} q_{\sigma} \Pi_{i}^{\{\rho, \sigma\}}-k_{i}^{\mu} \Pi_{i}^{\{\rho, \nu\}}-k_{i}^{\nu} \Pi_{i}^{\{\rho, \mu\}}+k_{i}^{\rho} \Pi_{i}^{\{\mu, \nu\}}\right) M_{n}\left(k_{i}\right)+\mathcal{O}\left(q^{2}\right)$,

which demonstrates that, when the soft state is a graviton or a dilaton, on-shell gauge invariance of the string amplitude implies a soft theorem at subsubleading order even when string corrections are taken into account. Finally we can rewrite this expression as:

$$
\left.M_{n+1}^{\mu \nu}\right|_{\alpha^{\prime}}=\frac{\alpha^{\prime}}{2} \sum_{i=1}^{n}\left(q_{\rho} k_{i}^{\mu} \eta_{\sigma}^{\nu}+q_{\sigma} k_{i}^{\nu} \eta_{\rho}^{\mu}-\left(k_{i} \cdot q\right) \eta_{\rho}^{\mu} \eta_{\sigma}^{\nu}-\frac{k_{i}^{\mu} k_{i}^{\nu}}{k_{i} \cdot q} q_{\rho} q_{\sigma}\right) \Pi_{i}^{\{\rho, \sigma\}} M_{n}\left(k_{i}\right)+\mathcal{O}\left(q^{2}\right) .
$$

This is exactly the expression we found in eq. (2.24) from explicit calculations. As we noticed in eq. (3.8), this expression vanishes when contracted with the polarization of the dilaton. For the graviton, we have explained that the term singular in $q \rightarrow 0$ comes from the exchange diagram where the the soft graviton scatters on an external massless closed string. When that string is a graviton, respectively, dilaton, the exchanged states is the opposite, i.e. a dilaton, respectively, graviton, meaning that the $n$-point amplitude in the soft theorem may involve other external hard states, than those specified in the $n+1$ point amplitude. The non-singular terms on the other hand are, as we have shown, direct consequences of on-shell gauge invariance.

For the sake of completeness we conclude this section by comparing to the low-energy effective action that appears in ref. [90], given by

$$
\begin{aligned}
S=\int d^{D} x \sqrt{-G} & \left\{\frac{1}{2 \kappa_{D}^{2}} R-\frac{1}{2} \partial_{\mu} \phi G^{\mu \nu} \partial_{\nu} \phi-\frac{1}{24 \kappa_{D}^{2}} \mathrm{e}^{-\frac{4 \kappa_{D} \phi}{\sqrt{D-2}}} H^{2}\right. \\
+ & \left.\frac{\alpha^{\prime}}{4} \mathrm{e}^{-\frac{2 \kappa_{D} \phi}{\sqrt{D-2}}}\left[\frac{1}{2 \kappa_{D}^{2}}\left(R_{\mu \nu \rho \sigma}^{2}-4 R_{\mu \nu}^{2}+R^{2}\right)+\cdots\right]+\mathcal{O}\left(\alpha^{\prime 2}\right)\right\},
\end{aligned}
$$

where, with respect to eq. (3.1) of ref. [90], we have chosen a different overall normalization and have introduced a canonically normalized dilaton. The first line corresponds to the field theory limit and from it we can reproduce the two first couplings in eq. (4.3) 
together with the field theoretical three-graviton amplitude. The second line shows the string corrections linear in $\alpha^{\prime}$, where we have only written down the terms that gives corrections to the three-point amplitude, while the ellipsis $\cdots$ denotes the higher-point operators. In particular, the last coupling in eq. (4.3) is reproduced by taking the lowest term of the Gauss-Bonnet part together with a dilaton coming from the exponential in front of the Gauss-Bonnet term, while the cubic term in the metric coming from only the Gauss-Bonnet part reproduces the first string correction to the three-graviton amplitude in eq. (4.2), as already noticed in ref. [92].

\section{Discussion and conclusion}

In this paper we completed the computation of the amplitude involving $n+1$ massless closed string states in the bosonic string to the subsubleading order in the soft momentum expansion of one of the external states. When the soft state is either a graviton or a dilaton we showed that the result can be expressed as a soft theorem to subsubleading order. The graviton soft theorem has string corrections at the subsubleading order in the bosonic string, while the string corrections for the dilaton all vanish. The leading and subleading terms were already obtained in ref. [78].

The calculation involves an extension of the technique developed in ref. [78], which is rather involved, but the final result has a very simple explanation: the basic ingredients to derive the soft-theorems are gauge invariance and the three-point amplitude with massless closed string states that, in the bosonic string, contains also string corrections. The procedure is an extension of the one followed in ref. [62], where the total amplitude is written as a sum of two types of terms. One corresponds to diagrams where the soft leg is attached to each of the other external legs through the three-point amplitude of the soft state and two other massless states. These terms trivially factorize and in general have a pole when the momentum of the soft state goes to zero. The other type of terms do not factorize trivially and are finite in the soft limit. The two types of contributions, however, are not separately gauge invariant. Thus, by imposing on-shell gauge invariance of the full amplitude, one is able to factorize the soft behavior of the total amplitude up to subsubleading order in the soft momentum. With respect to the procedure of ref. [62] for the case of a soft graviton, there are, however, two important differences. Gauge invariance imposed on the amplitude $M_{\mu \nu}$ does not only give the soft behavior for the graviton, when saturated with the graviton polarization $\epsilon_{g}^{\mu \nu}$, but also that for the dilaton when saturated with the polarization of the dilaton $\epsilon_{d}^{\mu \nu}$. The second difference with respect to the field theoretical behavior of an amplitude with only gravitons, is that, in general, the state exchanged in the propagator of the pole term is not necessarily the same that appears in the corresponding external leg. This happens in the string correction term in the amplitude with only gravitons and in the case of a soft dilaton.

We thus arrive to the conclusion that the soft theorems for the graviton and the dilaton are both consequence of gauge invariance and that the string corrections appearing at subsubleading order for the graviton are direct consequences of the three-point amplitude with massless closed strings having string corrections in the bosonic string. Since the three- 
point amplitude in superstring has no string corrections, we expect no string corrections in the soft behavior of a graviton in superstring.

A curious outcome of our results is that the soft theorem of a dilaton contains, at subleading order, the generator of scale transformation and, at subsubleading order, the generator of special conformal transformations as is also the case in the soft theorem of a Nambu-Goldstone boson of spontaneously broken conformal symmetry [24]. Some discussions of this was given in ref. [24], but it would be interesting to have a more physical understanding of why this happens. Finally, it would also be interesting to extend our considerations to the soft behavior of the Kalb-Ramond antisymmetric tensor, and furthermore how the results go over to the case of superstrings.

\section{Acknowledgments}

We thank Massimo Bianchi, Marco Billò, Marialuisa Frau, Andrea Guerrieri, Alberto Lerda, Carlo Maccaferri, Josh Nohle, Igor Pesando and Congkao Wen for many useful discussions. We owe special thanks to Josh Nohle for many relevant comments on our results.

\section{A Results of integrals}

All integrals necessary for the results of this paper can be reduced to the general form:

$$
I_{i_{1} i_{2} \ldots}^{j_{1} j_{2} \ldots}=\frac{1}{2 \pi} \int d^{2} z \frac{\prod_{l=1}^{n}\left|z-z_{l}\right|^{\alpha^{\prime} k_{l} q}}{\left(z-z_{i_{1}}\right)\left(z-z_{i_{2}}\right) \cdots\left(\bar{z}-\bar{z}_{j_{1}}\right)\left(\bar{z}-\bar{z}_{j_{2}}\right) \cdots} .
$$

We are using the convention $d^{2} z=2 d \operatorname{Re}(z) d \operatorname{Im}(z)$. The quantity $I_{j_{1} j_{2} \ldots}^{i_{1} i_{2} \ldots}$ is symmetric in its lower, respectively upper indices and complex conjugation exchanges the lower and upper indices. These integrals can generically be evaluated after an expansion in $q$ by a substitution of the form $z \rightarrow z_{i}+\rho e^{i \theta}$. It can be useful to substitute further $e^{i \theta} \rightarrow \omega$, such that $\int_{0}^{2 \pi} d \theta \cdots \rightarrow \oint d \omega \cdots$, enabling use of Cauchy's integral formula over the unit circle. To evaluate the integrals one must consider, case by case, when indices of $I_{j_{1} j_{2} \ldots}^{i_{1} i_{2} \ldots}$ are equal or not. Thus, in the following different labels on indices indicates the case when they are not equal to each other.

We have explicitly computed all integrals to the relevant order in $q$ involved in this paper using the mentioned procedure. Through this procedure, in ref. [78] the integrals $I_{i}^{i}$ and $I_{j}^{i}$ were already computed to the order $q^{1}$, with the results:

$$
\begin{aligned}
I_{i}^{i}= & \frac{2}{\alpha^{\prime} k_{i} q}\left(1+\alpha^{\prime} \sum_{j \neq i}\left(k_{j} q\right) \log \left|z_{i}-z_{j}\right|+\frac{\left(\alpha^{\prime}\right)^{2}}{2} \sum_{j \neq i} \sum_{k \neq i}\left(k_{j} q\right)\left(k_{k} q\right) \log \left|z_{i}-z_{j}\right| \log \left|z_{i}-z_{k}\right|\right) \\
& +\left(\alpha^{\prime}\right)^{2} \sum_{j \neq i}\left(k_{j} q\right) \log ^{2}\left|z_{i}-z_{j}\right|+\log \Lambda^{2}+\mathcal{O}\left(q^{2}\right) \\
I_{i}^{j}= & \sum_{m \neq i, j} \frac{\alpha^{\prime} q k_{m}}{2}\left(\operatorname{Li}_{2}\left(\frac{\bar{z}_{i}-\bar{z}_{m}}{\bar{z}_{i}-\bar{z}_{j}}\right)-\operatorname{Li}_{2}\left(\frac{z_{i}-z_{m}}{z_{i}-z_{j}}\right)-2 \log \frac{\bar{z}_{m}-\bar{z}_{j}}{\bar{z}_{i}-\bar{z}_{j}} \log \frac{\left|z_{i}-z_{j}\right|}{\left|z_{i}-z_{m}\right|}\right) \\
& -\log \left|z_{i}-z_{j}\right|^{2}+\log \Lambda^{2}+\mathcal{O}\left(q^{2}\right)
\end{aligned}
$$

where $\Lambda$ is a cutoff that vanishes, when inserted in the appropriate expression. 
It is useful to express the symmetrized and antisymmetrized form of latter expressions:

$$
\begin{aligned}
\frac{1}{2}\left(I_{i}^{j}+I_{j}^{i}\right)= & \log \Lambda^{2}-\log \left|z_{i}-z_{j}\right|^{2}+\sum_{m \neq i, j} \alpha^{\prime} q k_{m}\left(\log \left|z_{m}-z_{j}\right| \log \left|z_{i}-z_{m}\right|\right. \\
& \left.-\log \left|z_{m}-z_{j}\right| \log \left|z_{i}-z_{j}\right|-\log \left|z_{i}-z_{j}\right| \log \left|z_{i}-z_{m}\right|\right)+\mathcal{O}\left(q^{2}\right) \\
\frac{1}{2}\left(I_{i}^{j}-I_{j}^{i}\right)= & \frac{1}{2} \sum_{m \neq i} \alpha^{\prime} q \cdot k_{m} \operatorname{Li}_{2}\left(\frac{\bar{z}_{i}-\bar{z}_{m}}{\bar{z}_{i}-\bar{z}_{j}}\right)-\frac{1}{2} \sum_{m \neq i} \alpha^{\prime} q \cdot k_{m} \operatorname{Li}_{2}\left(\frac{z_{i}-z_{m}}{z_{i}-z_{j}}\right) \\
& +\frac{1}{2} \sum_{m \neq i, j} \alpha^{\prime} q \cdot k_{m} \log \frac{\left|z_{i}-z_{j}\right|}{\left|z_{i}-z_{m}\right|} \log \left[\frac{z_{m}-z_{j}}{\bar{z}_{m}-\bar{z}_{j}} \frac{\bar{z}_{i}-\bar{z}_{j}}{z_{i}-z_{j}}\right]+\mathcal{O}\left(q^{2}\right)
\end{aligned}
$$

It is a long and tedious exercise to evaluate all other integrals by the above mentioned procedure. In this appendix we therefore instead present an alternative shorter derivation leading to the same results, by making use of the above two expressions.

By using partial derivatives with respect to the $z_{i}$ 's it is possible to directly get the higher-index integrals from the two basic integrals above. The following four integrals for instance are readily derived in this way, and they will be used, together with the previous two integrals, as master integrals as will be explained below:

$$
\begin{aligned}
I_{i i}^{i}= & \frac{1}{1-\frac{\alpha^{\prime}}{2} k_{i} q} \partial_{z_{i}} I_{i}^{i}=\sum_{j \neq i}\left\{\frac{1}{z_{i}-z_{j}}\left[\frac{q k_{j}}{q k_{i}}+\alpha^{\prime} q \cdot k_{j} \log \left|z_{i}-z_{j}\right|+\frac{\alpha^{\prime}\left(q k_{j}\right)}{2}\right]\right. \\
& \left.+\frac{1}{2} \sum_{l \neq i} \frac{\left(\alpha^{\prime} q k_{j}\right)\left(\alpha^{\prime} q k_{l}\right)}{\alpha^{\prime} q k_{i}}\left[\frac{\log \left|z_{i}-z_{j}\right|}{z_{i}-z_{l}}+\frac{\log \left|z_{i}-z_{l}\right|}{z_{i}-z_{j}}\right]\right\}+\mathcal{O}\left(q^{2}\right) \\
I_{i i}^{j}= & \frac{1}{1-\frac{\alpha^{\prime}}{2}\left(k_{i} q\right)} \partial_{z_{i}} I_{i}^{j}=-\frac{1}{z_{i}-z_{j}}\left(1+\frac{\alpha^{\prime} q k_{i}}{2}+\alpha^{\prime}\left(q k_{i}+q k_{j}\right) \log \left|z_{i}-z_{j}\right|\right) \\
& +\sum_{l \neq i, j} \alpha^{\prime} q k_{l}\left\{\frac{\log \left|z_{j}-z_{l}\right|}{z_{i}-z_{l}}-\frac{\log \left|z_{i}-z_{j}\right|}{z_{i}-z_{l}}-\frac{\log \left|z_{j}-z_{l}\right|}{z_{i}-z_{j}}\right\}+\mathcal{O}\left(q^{2}\right) \\
I_{i i}^{i i}= & \frac{1}{1-\frac{\alpha^{\prime} q k_{i}}{2}} \partial_{\bar{z}_{i}} I_{i i}^{i} \\
= & \frac{1}{2} \sum_{j \neq i} \frac{\alpha^{\prime} q k_{j}}{\left|z_{i}-z_{j}\right|^{2}}\left(1+\frac{q k_{j}}{q k_{i}}+\frac{1}{2} \sum_{l \neq i, j} \frac{q k_{l}}{q k_{i}}\left[\frac{\bar{z}_{i}-\bar{z}_{j}}{\bar{z}_{i}-\bar{z}_{l}}+\frac{z_{i}-z_{j}}{z_{i}-z_{l}}\right]\right)+\mathcal{O}\left(q^{2}\right) \\
I_{i i}^{j j}= & \frac{1}{1-\frac{\alpha^{\prime} q k_{j}}{2}} \partial_{\bar{z}_{j}} I_{i i}^{j}=-\frac{1}{2\left|z_{i}-z_{j}\right|^{2}} \sum_{l \neq i, j} \alpha^{\prime} q k_{l} \frac{\left(\bar{z}_{i}-\bar{z}_{l}\right)\left(z_{j}-z_{l}\right)}{\left(z_{i}-z_{l}\right)\left(\bar{z}_{j}-\bar{z}_{l}\right)}+\mathcal{O}\left(q^{2}\right)
\end{aligned}
$$

All the other integrals relevant in the soft limit are related to the six master integrals by simple algebraic relations which follows from a useful rewriting of the holomorphic and anti-holomorphic poles of eq. (A.1). $I_{i_{1} i_{2} \ldots}^{j_{1} j_{2} \ldots}$ has poles both in $z=z_{i_{1}}, z=z_{i_{2}}$ and in $\bar{z}=\bar{z}_{j_{1}}$, 
$\bar{z}=\bar{z}_{j_{2}}$. It can be written as linear combination of the quantities $I_{i_{1} \ldots}^{j_{1} \ldots}, I_{i_{1} \ldots}^{j_{2} \ldots}, I_{i_{2} \ldots}^{j_{1} \ldots} I_{i_{2} \ldots}^{j_{2} \ldots}$ having only two of the above mentioned poles. This follows from the identity:

$$
\frac{1}{\left(z-z_{i_{1}}\right)\left(z-z_{i_{2}}\right)}=\frac{1}{\left(z_{i_{1}}-z_{i_{2}}\right)}\left[\frac{1}{\left(z-z_{i_{1}}\right)}-\frac{1}{\left(z-z_{i_{2}}\right)}\right]
$$

with a similar relation for the $\bar{z}$ variable. This determines:

$$
I_{i_{1} i_{2} \ldots}^{j_{1} j_{2} \ldots}=\frac{I_{i_{1} \ldots}^{j_{1} j_{2} \ldots}-I_{i_{2} \ldots}^{j_{1} j_{2} \ldots}}{\left(z_{i_{1}}-z_{i_{2}}\right)}=\frac{I_{i_{1} \ldots}^{j_{1} \ldots}-I_{i_{1} \ldots}^{j_{2} \ldots}-I_{i_{2} \ldots}^{j_{1} \ldots}+I_{i_{2} \ldots}^{j_{2} \ldots}}{\left(z_{i_{1}}-z_{i_{2}}\right)\left(\bar{z}_{j_{1}}-\bar{z}_{j_{2}}\right)}
$$

By applying eq. (A.11) iteratively, all the necessary integrals can be computed. Following such an analysis, we present in the subsequent sections the results of all integrals involved in this work to the necessary order in $q$. It will be useful to notice from the above decomposition that an integral $I_{i_{1} i_{2} \ldots}^{j_{1} j_{2} \ldots}$ can yield a term of order $q^{-1}$ only if one of its lower indices is equal to one of the upper ones, since its decomposition may only then contain $I_{i}^{i}$. This property will be used throughout our derivations.

Integrals of the form $\boldsymbol{I}_{\boldsymbol{i m}}^{\boldsymbol{j} \boldsymbol{n}}$. The integrals $I_{i i}^{i i}$ and $I_{i i}^{j j}$ were already given above in eqs. (A.8)-(A.9).

Next, according to eq. (A.11) we have:

$$
I_{i i}^{i j}=\frac{I_{i i}^{i}-I_{i i}^{j}}{\left(\bar{z}_{i}-\bar{z}_{j}\right)}=\frac{1}{\left|z_{i}-z_{j}\right|^{2}}\left(1+\frac{q k_{j}}{q k_{i}}+\sum_{l \neq i, j} \frac{q k_{l}}{q k_{i}} \frac{z_{i}-z_{j}}{z_{i}-z_{l}}\right)+\mathcal{O}(q)
$$

The quantities $I_{i i}^{i}$ and $I_{i i}^{j}$ have been computed up to the order $q$ and therefore we could give $I_{i i}^{i j}$ up to this order in the soft expansion. However, this integral appears in $S_{1}$ and $S_{3}$ multiplied by a factor $q$, therefore, only the terms of order $q^{0}$ are relevant. This kind of restriction will be implicit in all the following results.

Then,

$$
\begin{aligned}
I_{i i}^{j m} & =\frac{I_{i i}^{j}-I_{i i}^{m}}{\bar{z}_{i}-\bar{z}_{m}}=\frac{1}{\bar{z}_{j}-\bar{z}_{m}}\left(\frac{1}{z_{i}-z_{m}}-\frac{1}{z_{i}-z_{j}}\right)+\mathcal{O}(q) \\
I_{i m}^{i m} & =\frac{I_{i}^{i}-I_{i}^{m}-I_{m}^{i}+I_{m}^{m}}{\left|z_{i}-z_{m}\right|^{2}}=\frac{2}{\left|z_{i}-z_{m}\right|^{2}}\left(\frac{1}{\alpha^{\prime} q k_{i}}+\frac{1}{\alpha^{\prime} q k_{m}}\right)+\mathcal{O}\left(q^{0}\right) \\
I_{i m}^{i n} & =\frac{I_{i}^{i}-I_{i}^{n}-I_{m}^{i}+I_{m}^{n}}{\left(z_{i}-z_{m}\right)\left(\bar{z}_{i}-\bar{z}_{n}\right)}=\frac{2}{\alpha^{\prime} q k_{i}} \frac{1}{\left(z_{i}-z_{m}\right)\left(\bar{z}_{i}-\bar{z}_{n}\right)}+O\left(q^{0}\right) \\
I_{i m}^{j n}= & \frac{I_{i}^{j}-I_{i}^{n}-I_{m}^{j}+I_{m}^{n}}{\left(z_{i}-z_{m}\right)\left(\bar{z}_{j}-\bar{z}_{n}\right)}=0+\mathcal{O}\left(q^{0}\right)
\end{aligned}
$$

As we have already noticed, the expansion of the integrals may start from order $q^{-1}$ only if one of the upper indices is equal to one of the lower ones. Thus the result of eq. (A.16) could have been readily inferred from this property, without making the intermediate decomposition. This example serves as a demonstration of this property, which we will directly use for the rest of this appendix. 
Integrals of the form $\boldsymbol{I}_{\boldsymbol{i m n}}^{j}$. Using eq. (A.11) iteratively, we get

$$
\begin{aligned}
I_{i i m}^{i}= & \frac{I_{i i}^{i}}{z_{i}-z_{m}}-\frac{I_{i}^{i}-I_{m}^{i}}{\left(z_{i}-z_{m}\right)^{2}}=-\frac{2}{\left(z_{i}-z_{m}\right)^{2}} \\
& \times\left[\frac{1}{\alpha^{\prime} q k_{i}}+\log \left|z_{i}-z_{m}\right|+\sum_{l \neq i} \frac{q k_{l}}{q k_{i}}\left(\log \left|z_{i}-z_{l}\right|-\frac{1}{2} \frac{z_{i}-z_{m}}{z_{i}-z_{l}}\right)\right]+\mathcal{O}(q) \\
I_{i i j}^{j}= & \frac{I_{i i}^{j}}{z_{i}-z_{j}}-\frac{I_{i}^{j}-I_{j}^{j}}{\left(z_{i}-z_{j}\right)^{2}} \\
= & \frac{2}{\left(z_{i}-z_{j}\right)^{2}}\left[\frac{1}{\alpha^{\prime} q k_{j}}-\frac{1}{2}+\log \left|z_{i}-z_{j}\right|+\sum_{l \neq j} \frac{q k_{l}}{q k_{j}} \log \left|z_{l}-z_{j}\right|\right]+\mathcal{O}(q) \\
I_{i m n}^{i}= & \frac{1}{z_{m}-z_{n}}\left(\frac{I_{i}^{i}-I_{m}^{i}}{z_{i}-z_{m}}-\frac{I_{i}^{i}-I_{n}^{i}}{z_{i}-z_{n}}\right)=\frac{2}{\alpha^{\prime} q k_{i}} \frac{1}{\left(z_{i}-z_{m}\right)\left(z_{i}-z_{n}\right)}+\mathcal{O}\left(q^{0}\right) \\
I_{i i m}^{j}= & \frac{I_{i i}^{j}}{z_{i}-z_{m}}-\frac{I_{i}^{j}-I_{m}^{j}}{\left(z_{i}-z_{m}\right)^{2}} \\
= & -\frac{1}{\left(z_{i}-z_{m}\right)\left(z_{i}-z_{j}\right)}+\frac{2}{\left(z_{i}-z_{m}\right)^{2}}\left(\log \left|z_{i}-z_{j}\right|-\log \left|z_{j}-z_{m}\right|\right)+\mathcal{O}(q) \\
I_{i m n}^{j}= & 0+\mathcal{O}\left(q^{0}\right)
\end{aligned}
$$

The result of the last integral follows immediately due to non of the upper and lower indices being pairwise equal.

Integrals of the form $\boldsymbol{I}_{\boldsymbol{i m n}}^{\boldsymbol{j l} \text {. }}$ Using eq. (A.11) iteratively, and the identities $I_{m}^{i i}=\left(I_{i i}^{m}\right)^{*}$ and $I_{i}^{i i}=\left(I_{i i}^{i}\right)^{*}$, where $*$ denotes the complex conjugate, we get

$$
\begin{aligned}
I_{i i m}^{i i} & =\frac{I_{i i}^{i i}}{z_{i}-z_{m}}-\frac{\left(I_{i i}^{i}\right)^{*}-\left(I_{i i}^{m}\right)^{*}}{\left(z_{i}-z_{m}\right)^{2}} \\
& =-\frac{1}{\left|z_{i}-z_{m}\right|^{2}\left(z_{i}-z_{m}\right)}\left(1+\frac{q k_{m}}{q k_{i}}+\sum_{l \neq i, m} \frac{q k_{l}}{q k_{i}} \frac{\bar{z}_{i}-\bar{z}_{m}}{\bar{z}_{i}-\bar{z}_{l}}\right)+\mathcal{O}(q) \\
I_{i i j}^{j j} & =\frac{I_{i i}^{j j}}{z_{i}-z_{j}}-\frac{I_{i}^{j j}-I_{j}^{j j}}{\left(z_{i}-z_{j}\right)^{2}} \\
& =-\frac{1}{\left|z_{i}-z_{j}\right|^{2}\left(z_{i}-z_{j}\right)}\left(1+\frac{q k_{i}}{q k_{j}}+\sum_{l \neq i, j} \frac{q k_{l}}{q k_{j}} \frac{\bar{z}_{j}-\bar{z}_{i}}{\bar{z}_{j}-\bar{z}_{l}}\right)+\mathcal{O}(q) \\
I_{i i m}^{j j} & =-\frac{I_{i i}^{j j}}{z_{i}-z_{m}}-\frac{I_{i}^{j j}-I_{m}^{j j}}{\left(z_{i}-z_{m}\right)^{2}}=-\frac{\left.\bar{z}_{i}\right)}{\left(\bar{z}_{i}-\bar{z}_{j}\right)\left(z_{i}-z_{m}\right)^{2}\left(\bar{z}_{j}-\bar{z}_{m}\right)}+\mathcal{O}(q) \\
I_{i i j}^{i j} & =\frac{I_{i i}^{i}-I_{i i}^{j}}{\left|z_{i}-z_{j}\right|^{2}}-\frac{I_{i}^{i}-I_{j}^{i}-I_{i}^{j}+I_{j}^{j}}{\left(z_{i}-z_{j}\right)\left|z_{i}-z_{j}\right|^{2}}
\end{aligned}
$$




$$
\begin{aligned}
& =-\frac{2}{\left(z_{i}-z_{j}\right)\left|z_{i}-z_{j}\right|^{2}}\left(\frac{1}{\alpha^{\prime} q k_{i}}+\frac{1}{\alpha^{\prime} q k_{j}}\right)+\mathcal{O}\left(q^{0}\right) \\
I_{i i k}^{i m} & =\frac{I_{i i}^{i}-I_{i i}^{m}}{\left(z_{i}-z_{k}\right)\left(\bar{z}_{i}-\bar{z}_{m}\right)}-\frac{I_{i}^{i}-I_{k}^{i}+I_{i}^{m}-I_{k}^{m}}{\left(z_{i}-z_{k}\right)^{2}\left(\bar{z}_{i}-\bar{z}_{m}\right)} \\
& =-\frac{2}{\left(z_{i}-z_{k}\right)^{2}\left(\bar{z}_{i}-\bar{z}_{m}\right) \alpha^{\prime} q k_{i}}+\mathcal{O}\left(q^{0}\right) \\
I_{i i j}^{j l} & =\frac{I_{i i}^{j}-I_{i i}^{l}}{\left(z_{i}-z_{j}\right)\left(\bar{z}_{j}-\bar{z}_{l}\right)}-\frac{I_{i}^{j}-I_{j}^{j}+I_{i}^{l}-I_{j}^{l}}{\left(z_{i}-z_{j}\right)^{2}\left(\bar{z}_{j}-\bar{z}_{l}\right)}=\frac{2}{\alpha^{\prime} q k_{j}} \frac{1}{\left(z_{i}-z_{j}\right)^{2}\left(\bar{z}_{j}-\bar{z}_{l}\right)}+\mathcal{O}\left(q^{0}\right)
\end{aligned}
$$

Finally, the integrals $I_{i i m}^{j n}, I_{i m n}^{i i}, I_{i m n}^{j j}$ have either all the upper indices different from the lower ones or more than one lower index equal to an upper one. Thus according to the general property, noticed specifically after eq. (A.16), we have:

$$
I_{i i m}^{j n}=I_{i m n}^{i i}=I_{i m n}^{j j}=0+\mathcal{O}\left(q^{0}\right)
$$

Integrals of the form $\boldsymbol{I}_{\text {iimn }}^{j}$. Using eq. (A.11) iteratively,

$$
\begin{aligned}
I_{i i k m}^{i} & =\frac{1}{\left(z_{k}-z_{m}\right)\left(z_{i}-z_{k}\right)}\left(I_{i i}^{i}-\frac{I_{i}^{i}-I_{k}^{i}}{\left(z_{i}-z_{k}\right)}\right)-\frac{1}{\left(z_{i}-z_{m}\right)\left(z_{k}-z_{m}\right)}\left(1-\frac{I_{i}^{i}-I_{m}^{i}}{\left(z_{i}-z_{m}\right)}\right) \\
& =\frac{2}{\alpha^{\prime} q k_{i}} \frac{1}{\left(z_{m}-z_{k}\right)}\left[\frac{1}{\left(z_{i}-z_{k}\right)^{2}}-\frac{1}{\left(z_{i}-z_{m}\right)^{2}}\right]+\mathcal{O}\left(q^{0}\right) \\
I_{i i j l}^{j} & =\frac{I_{i i}^{j}}{\left(z_{i}-z_{l}\right)\left(z_{i}-z_{j}\right)}-\frac{I_{i}^{j}-I_{j}^{j}}{\left(z_{j}-z_{l}\right)\left(z_{i}-z_{j}\right)^{2}}-\frac{I_{i i}^{j}}{\left(z_{j}-z_{l}\right)\left(z_{i}-z_{l}\right)}+\frac{I_{i}^{j}-I_{l}^{j}}{\left(z_{j}-z_{l}\right)\left(z_{i}-z_{l}\right)^{2}} \\
& =\frac{2}{\alpha^{\prime} q k_{j}} \frac{1}{\left(z_{j}-z_{i}\right)^{2}\left(z_{j}-z_{l}\right)}+\mathcal{O}\left(q^{0}\right) \\
I_{\text {iimn }}^{j} & =0+\mathcal{O}\left(q^{0}\right)
\end{aligned}
$$

The last result follows since none of the lower indices are equal to the upper one.

Integrals of the form $\boldsymbol{I}_{\boldsymbol{i m n}}^{\boldsymbol{j} \boldsymbol{j} \boldsymbol{n}}$. Using eq. (A.11) iteratively,

$$
\begin{aligned}
I_{i i k}^{i i k} & =\frac{I_{i i}^{i i}}{\left|z_{i}-z_{k}\right|^{2}}-\frac{I_{i i}^{i}-I_{i i}^{k}}{\left|z_{i}-z_{k}\right|^{2}\left(\bar{z}_{i}-\bar{z}_{k}\right)}-\frac{I_{i}^{i i}-I_{k}^{i i}}{\left(z_{i}-z_{k}\right)\left|z_{i}-z_{k}\right|^{2}}+\frac{I_{i}^{i}-I_{i}^{k}-I_{k}^{i}+I_{k}^{k}}{\left|z_{i}-z_{k}\right|^{4}} \\
& =\frac{2}{\left|z_{i}-z_{k}\right|^{4}}\left(\frac{1}{\alpha^{\prime} q k_{i}}+\frac{1}{\alpha^{\prime} q k_{k}}\right)+\mathcal{O}\left(q^{0}\right) \\
I_{i i k}^{i i m} & =\frac{I_{i i}^{i i}}{\left(z_{i}-z_{k}\right)\left(\bar{z}_{i}-\bar{z}_{m}\right)}-\frac{I_{i i}^{i}-I_{i i}^{m}}{\left(z_{i}-z_{k}\right)\left(\bar{z}_{i}-\bar{z}_{m}\right)^{2}}-\frac{I_{i}^{i i}-I_{k}^{i i}}{\left(z_{i}-z_{k}\right)^{2}\left(\bar{z}_{i}-\bar{z}_{m}\right)}+\frac{I_{i}^{i}-I_{k}^{i}-I_{i}^{m}+I_{k}^{m}}{\left(z_{i}-z_{k}\right)^{2}\left(\bar{z}_{i}-\bar{z}_{m}\right)^{2}} \\
& =\frac{2}{\alpha^{\prime} q k_{i}\left(z_{i}-z_{k}\right)^{2}\left(\bar{z}_{i}-\bar{z}_{m}\right)^{2}}+\mathcal{O}\left(q^{0}\right) \\
I_{i i j}^{j j i} & =\frac{I_{i i}^{j j}}{\left|z_{i}-z_{j}\right|^{2}}-\frac{I_{i i}^{j}-I_{i i}^{i}}{\left|z_{i}-z_{j}\right|^{4}\left(\bar{z}_{i}-\bar{z}_{j}\right)}-\frac{I_{i}^{j j}-I_{j}^{j j}}{\left|z_{i}-z_{j}\right|^{2}\left(z_{i}-z_{j}\right)}+\frac{I_{i}^{j}-I_{i}^{i}-I_{j}^{j}+I_{j}^{i}}{\left|z_{i}-z_{j}\right|^{4}} \\
& =-\frac{2}{\left|z_{i}-z_{j}\right|^{4}}\left(\frac{1}{\alpha^{\prime} q k_{i}}+\frac{1}{\alpha^{\prime} q k_{j}}\right)+\mathcal{O}\left(q^{0}\right) \\
I_{i i k}^{j j k} & =\frac{I_{i i}^{j j}}{\left(z_{i}-z_{k}\right)\left(\bar{z}_{j}-\bar{z}_{k}\right)}-\frac{I_{i i}^{j j}-I_{i i}^{k}}{\left(z_{i}-z_{k}\right)\left(\bar{z}_{j}-\bar{z}_{k}\right)^{2}}-\frac{I_{i}^{j j}}{\left(z_{i}-z_{k}\right)^{2}\left(\bar{z}_{j}-\bar{z}_{k}\right)}+\frac{I_{i}^{j}-I_{k}^{j}-I_{i}^{k}+I_{k}^{k}}{\left(z_{i}-z_{k}\right)^{2}\left(\bar{z}_{j}-\bar{z}_{k}\right)^{2}}
\end{aligned}
$$




$$
\begin{aligned}
& =\frac{2}{\left(\bar{z}_{k}-\bar{z}_{j}\right)^{2}\left(z_{k}-z_{i}\right)^{2}} \frac{1}{\alpha^{\prime} q k_{k}}+\mathcal{O}\left(q^{0}\right) \\
I_{i i k}^{j j i} & =\frac{I_{i i}^{j j}}{\left(z_{i}-z_{k}\right)\left(\bar{z}_{j}-\bar{z}_{i}\right)}-\frac{I_{i i}^{j}-I_{i i}^{i}}{\left(z_{i}-z_{k}\right)\left(\bar{z}_{j}-\bar{z}_{i}\right)^{2}}-\frac{I_{i}^{j j}-I_{k}^{j j}}{\left(z_{i}-z_{k}\right)^{2}\left(\bar{z}_{j}-\bar{z}_{i}\right)}+\frac{I_{i}^{j}-I_{k}^{j}-I_{i}^{i}+I_{k}^{i}}{\left(z_{i}-z_{k}\right)^{2}\left(\bar{z}_{j}-\bar{z}_{i}\right)^{2}} \\
& =-\frac{2}{\left(z_{i}-z_{k}\right)^{2}\left(\bar{z}_{i}-\bar{z}_{j}\right)^{2} \alpha^{\prime} q k_{i}}+\mathcal{O}\left(q^{0}\right) \\
I_{i i m}^{j j n} & =0+\mathcal{O}\left(q^{0}\right)
\end{aligned}
$$

The last result follows since none of the lower indices are equal to the upper ones.

Integrals of the form $\boldsymbol{I}_{\boldsymbol{i i m n}}^{\boldsymbol{j} \boldsymbol{j}}$. For these integrals either two upper indices are equal to some lower indices, or none are equal. Thus neither of the cases contains $I_{i}^{i}$ in its decomposition and thus in any case,

$$
I_{\text {iimn }}^{i i}=I_{i i j m}^{j j}=I_{i i m n}^{j j}=0+\mathcal{O}\left(q^{0}\right)
$$

\section{B Matching with the subsubleading soft operator}

In this appendix we explicitly show how our main result in eq. (2.24) reproduces our explicit calculation of the amplitude of $n+1$ massless closed states in the bosonic string, presented in section 2 and appendix A, when the soft state is a graviton or a dilaton.

In eq. (2.24), the subsubleading soft operator is the sum of four parts which act on the $n$-point amplitude. The result of this action is given below and compared with explicit results in eqs. (2.10)-(2.12).

The action of the first term in eq. (2.24) on the $n$-point amplitude is:

$$
-\sum_{i=1}^{n} \frac{q_{\rho} J_{i}^{\mu \rho} q_{\sigma} J_{i}^{\nu \sigma}}{2 k_{i} q} M_{n}=M_{n} *\left[A_{\left(\theta^{0}\right)}^{\mu \nu}+A_{(\theta)}^{\mu \nu}+A_{\left(\theta^{2}\right)}^{\mu \nu}+A_{(\theta \bar{\theta})}^{\mu \nu}+A_{\left(\theta^{3}\right)}^{\mu \nu}+A_{\left(\theta^{2} \bar{\theta}\right)}^{\mu \nu}+A_{\left(\theta^{2} \bar{\theta}^{2}\right)}^{\mu \nu}\right]+c . c .
$$

We are denoting with $A_{\left(\theta^{\alpha} \bar{\theta}^{\beta}\right)}^{\mu \nu}, \alpha, \beta=0,1,2$, terms with different powers in the Grassmann variables $\left(\theta_{i}, \bar{\theta}_{j}\right)$.

The action of the second operator in eq. (2.24) gives:

$$
\begin{aligned}
& -\frac{1}{2} \sum_{i=1}^{n}\left(\frac{k_{i}^{\nu} q^{\mu}}{k_{i} q} q^{\sigma}+q^{\nu} \eta^{\mu \sigma}-\eta^{\nu \mu} q^{\sigma}\right) \frac{\partial}{\partial k_{i}^{\sigma}} M_{n}=-M_{n} *\left[\frac { 1 } { 2 } \sqrt { \frac { \alpha ^ { \prime } } { 2 } } \sum _ { i \neq j } \left(\frac{k_{i}^{\nu} q^{\mu}\left(k_{j} q\right)}{k_{i} q} \frac{\left(\theta_{j} \epsilon_{j} q\right)}{z_{j}-z_{i}}\right.\right. \\
& \left.\left.+\frac{q^{\nu} \theta_{j} \epsilon_{j}^{\mu}}{z_{j}-z_{i}}-\frac{\eta^{\mu \nu}\left(\theta_{j} \epsilon_{j} q\right)}{z_{j}-z_{i}}+c . c .\right)-\frac{\alpha^{\prime}}{2} \sum_{i \neq j}\left(k_{i}^{\mu} q^{\nu} \frac{k_{j} q}{k_{i} q}+q^{\nu} k_{j}^{\mu}-\eta^{\mu \nu} q k_{j}\right) \log \left|z_{i}-z_{j}\right|\right] .
\end{aligned}
$$

The action of the operators in the second and third line of eq. (2.24) will be considered in the end.

We restrict our analysis to the case in which the external soft particle is a graviton or a dilaton, meaning that the expressions above are projected on the symmetric combination of $\mu \nu$. All $A_{\left(\theta^{\alpha} \bar{\theta}^{\beta}\right)}^{\mu \nu}$ depend on the polarizations and momenta of the hard particles. Their explicit expressions are rather lengthy, but we may express them in more compact terms by using the explicit expressions for the quantities $I_{i_{1} i_{2} \ldots}^{j_{1} j_{2} \ldots}$ given in appendix A, which 
also allows for an easier identification with the expression in eqs. (2.10)-(2.12). The first quantity can be expressed as:

$$
A_{\left(\theta^{0}\right)}^{\mu \nu}=\frac{\alpha^{\prime}}{4}\left[\sum_{i \neq j}\left(k_{i}^{\nu} q^{\mu} \frac{\left(k_{j} q\right)}{k_{i} q}-\eta^{\mu \nu}\left(k_{j} q\right)+k_{j}^{\mu} q^{\nu}\right) \log \left|z_{i}-z_{j}\right|+\sum_{i, j=1}^{n} k_{i}^{(\mu} k_{j}^{\nu)} I^{(1)}{ }_{i}^{j}\right]
$$

with $k_{i}^{\{\mu} k_{j}^{\nu\}}=\frac{1}{2}\left(k_{i}^{\mu} k_{j}^{\nu}+k_{i}^{\nu} k_{j}^{\mu}\right)$. This expression is real and thus the c.c. terms simply give a factor of two. Because of this factor 2, the term with $\log \left|z_{i}-z_{j}\right|$ in eq. (B.3) cancel the analogous term in eq. (B.2). The remaining term in eq. (B.3) involving $I_{i}^{j}$ coincides with the symmetric part of $S_{1}^{(0)}$ in eq. (2.10a).

The next term reads:

$$
\begin{aligned}
& A_{(\theta)}^{\mu \nu}=\sqrt{\frac{\alpha^{\prime}}{2}}\left\{\frac { 1 } { 2 } \sum _ { i \neq j } \left[\left(\eta^{\mu \nu}-\frac{k_{i}^{\nu} q^{\mu}}{k_{i} q}\right) \frac{\left(\theta_{j} \epsilon_{j} q\right)}{z_{i}-z_{j}}-\frac{\theta_{j} \epsilon_{j}^{\mu} q^{\nu}}{z_{i}-z_{j}}\right.\right. \\
& \left.+\frac{k_{j} q}{k_{i} q} \frac{\theta_{i} \epsilon_{i}^{\nu} q^{\mu}}{z_{i}-z_{j}}+\left(\frac{k_{j}^{\mu} q^{\nu}}{q k_{i}}-\eta^{\mu \nu} \frac{k_{j} q}{k_{i} q}\right) \frac{\theta_{i} \epsilon_{i} q}{z_{i}-z_{j}}\right]+\frac{\alpha^{\prime}}{2} \sum_{i \neq j}\left[\frac{\left(k_{i} q\right) \theta_{i} \epsilon_{i}^{\{\mu} k_{j}^{\nu\}}}{z_{i}-z_{j}}-\frac{\left(k_{j} q\right) \theta_{i} \epsilon_{i}^{\{\mu} k_{i}^{\nu\}}}{z_{i}-z_{j}}\right] \\
& -\frac{\alpha^{\prime}}{2} \sum_{i \neq j} k_{i}^{\{\mu} k_{i}^{\nu\}}\left(\theta_{j} \epsilon_{j} q\right) I^{(0)}{ }_{i j}^{i}-\frac{\alpha^{\prime}}{2} \sum_{i \neq j} k_{i}^{\{\mu} k_{j}^{\nu\}} I^{(0)}{ }_{i j}^{j}\left(\theta_{j} \epsilon_{j} q\right)-\frac{\alpha^{\prime}}{2} \sum_{i \neq j \neq l} k_{i}^{\{\mu} k_{i}^{\nu\}}\left(\theta_{l} \epsilon_{l} q\right) I^{(0)}{ }_{i l}^{j} \\
& \left.+\sum_{i, j=1}^{n} \theta_{i} \epsilon_{i}^{\{\mu} k_{i}^{\nu\}} I^{(1)^{j}}{ }_{i i}\right\}
\end{aligned}
$$

The first three terms and their complex conjugate cancel the first three terms in eq. (B.2) and their complex conjugate, coming from the second soft operator. The third line of this expression makes up part of $S_{1}^{(1)}$ when the soft state is taken to be symmetrically polarized, i.e $\epsilon_{q \mu} \bar{\epsilon}_{q \nu} \rightarrow \epsilon_{q \mu \nu}^{S}$. The following terms of $S_{1}^{(1)}$ then remain to be matched:

$$
-\left(\frac{\alpha^{\prime}}{2}\right)^{\frac{3}{2}} \sum_{i \neq j}\left(\epsilon_{q} k_{i}\right)\left(\bar{\epsilon}_{q} k_{i}\right)\left(\theta_{i} \epsilon_{i} q\right) I^{(0)}{ }_{i i}^{i}-\left(\frac{\alpha^{\prime}}{2}\right)^{\frac{3}{2}} \sum_{i \neq j}\left(\epsilon_{q} k_{i}\right)\left(\bar{\epsilon}_{q} k_{j}\right)\left(\theta_{i} \epsilon_{i} q\right) I^{(0)^{j}}{ }_{i i}
$$

The last term in eq. (B.4) is equal to $S_{3}^{(0)}$ when the soft state again is a graviton or a dilaton. Finally, the second line does not match any term in $S_{1}, S_{2}$ and $S_{3}$, but they will be cancelled by the additional soft operators, discussed in the end, which at the same time will also produce the missing terms in eq. (B.5).

Next, we consider

$$
\begin{aligned}
& A_{\left(\theta^{2}\right)}^{\mu \nu}=\frac{1}{2} \sum_{i \neq j}\left[\frac{\theta_{i} \epsilon_{i}^{\nu} q^{\mu}\left(\theta_{j} \epsilon_{j} q\right)-\left(\eta^{\mu \nu}\left(\theta_{j} \epsilon_{j} q\right)-q^{\nu} \theta_{j} \epsilon_{j}^{\mu}\right)\left(\theta_{i} \epsilon_{i} q\right)}{q k_{i}\left(z_{i}-z_{j}\right)^{2}}\right] \\
& +\left(\frac{\alpha^{\prime}}{2}\right)^{2} \sum_{i \neq j} \frac{k_{i}^{\{\mu} k_{j}^{\nu\}}\left(\theta_{i} \epsilon_{i} q\right)\left(\theta_{j} \epsilon_{j} q\right)}{\left(z_{i}-z_{j}\right)^{2}} I^{(-1)_{j}^{j}}+\frac{1}{2}\left(\frac{\alpha^{\prime}}{2}\right)^{2} \sum_{i \neq j} k_{i}^{\mu} k_{i}^{\nu} \sum_{l \neq i, j} \frac{\left(q \theta_{j} \epsilon_{j}\right)\left(q \theta_{l} \epsilon_{l}\right) I^{(-1)_{i}^{i}}}{\left(z_{i}-z_{j}\right)\left(z_{i}-z_{l}\right)} \\
& -\left(\frac{\alpha^{\prime}}{2}\right)^{2} \sum_{i \neq j} k_{i}^{\{\nu} k_{j}^{\mu\}} \sum_{l \neq i, j} \frac{\left(q \theta_{j} \epsilon_{j}\right)\left(q \theta_{l} \epsilon_{l}\right) I^{(-1)^{j}}}{\left(z_{i}-z_{j}\right)\left(z_{j}-z_{l}\right)}-\frac{\alpha^{\prime}}{2} \sum_{i \neq j} \theta_{i} \epsilon_{i}^{\{\mu} k_{i}^{\nu\}}\left(q \theta_{j} \epsilon_{j}\right) I^{(0)}{ }_{i i j}^{i}
\end{aligned}
$$




$$
\begin{aligned}
& -\frac{\alpha^{\prime}}{2} \sum_{i \neq j} \theta_{i} \epsilon_{i}^{\{\mu} k_{j}^{\nu\}}\left(q \theta_{j} \epsilon_{j}\right) I^{(0)}{ }_{i i j}^{j}-\frac{\alpha^{\prime}}{2} \sum_{i \neq j \neq l} \theta_{i} \epsilon_{i}^{\{\mu} k_{j}^{\nu\}}\left(q \theta_{l} \epsilon_{l}\right) I_{i i l}^{(0)}{ }_{i i l}^{j} \\
& +\left(\frac{\alpha^{\prime}}{2}\right) \sum_{i \neq j} \frac{\theta_{i} \epsilon_{i}^{\{\mu}\left(k_{i}^{\nu\}}+k_{j}^{\nu\}}\right)\left(q \theta_{j} \epsilon_{j}\right)}{\left(z_{i}-z_{j}\right)} I_{i i}^{(0)^{j}}+\left(\frac{\alpha^{\prime}}{2}\right) \sum_{i \neq j} \frac{\left(q k_{j}\right) \theta_{j} \epsilon_{j}^{\{\nu} \theta_{i} \epsilon_{i}^{\mu\}}}{\left(z_{i}-z_{j}\right)^{2}}
\end{aligned}
$$

For the terms involving $I_{i}^{j}$ we can show that they are matched by part of the first term in $S_{1}^{(2)}$, by using the following identities:

$$
\begin{aligned}
& I_{i i j}^{j}=I_{i j i}^{j}=\frac{1}{\left(z_{i}-z_{j}\right)}\left[I_{i i}^{j}-\frac{I_{i}^{j}-I_{j}^{j}}{z_{i}-z_{j}}\right]=\frac{I^{(-1)_{j}^{j}}}{\left(z_{i}-z_{j}\right)^{2}}+O\left(q^{0}\right) \\
& I_{i l j}^{i}=\frac{1}{z_{l}-z_{j}}\left[\frac{I_{i}^{i}-I_{l}^{i}}{z_{i}-z_{l}}-\frac{I_{i}^{i}-I_{j}^{i}}{z_{i}-z_{j}}\right]=\frac{I^{(-1)_{i}^{i}}}{\left(z_{i}-z_{j}\right)\left(z_{i}-z_{l}\right)}+O\left(q^{0}\right) \\
& I_{i j l}^{j}=I_{i l j}^{j}=\frac{1}{z_{j}-z_{l}}\left[\frac{I_{i}^{j}-I_{j}^{j}}{z_{i}-z_{j}}-\frac{I_{i}^{j}-I_{l}^{j}}{z_{i}-z_{l}}\right]=-\frac{I^{(-1)_{j}^{j}}}{\left(z_{j}-z_{l}\right)\left(z_{i}-z_{j}\right)}+O\left(q^{0}\right) .
\end{aligned}
$$

The first term of $S_{1}^{(2)}$ furthermore contains the following term, unmatched by eq. (B.6):

$$
\left(\frac{\alpha^{\prime}}{2}\right)^{2} \sum_{i \neq j}\left(\epsilon_{q} k_{i}\right)\left(\bar{\epsilon}_{q} k_{i}\right)\left(\theta_{i} \epsilon_{i} q\right)\left(\theta_{j} \epsilon_{j} q\right) I_{i i j}^{(-1)^{i}}=-\left(\frac{\alpha^{\prime}}{2}\right)^{2} \sum_{i \neq j} \frac{\left(\epsilon_{q} k_{i}\right)\left(\bar{\epsilon}_{q} k_{i}\right)\left(\theta_{i} \epsilon_{i} q\right)\left(\theta_{j} \epsilon_{j} q\right)}{\left(z_{i}-z_{j}\right)^{2}} I_{i}^{(-1)^{i}}
$$

The terms involving $I_{i i l}^{j}$ in eq. (B.6) trivially reproduce the first part of $S_{3}^{(1)}$. Finally, the first and the last line in eq. (B.6) are not present in $S_{1}, S_{2}$ and $S_{3}$. Again, we discuss on the missing pieces in the end.

Next, we consider

$$
\begin{aligned}
& A_{\theta \bar{\theta}}^{\mu \nu}=\frac{1}{2}\left(\frac{\alpha^{\prime}}{2}\right)^{2} \sum_{i \neq j} \frac{\left(k_{i}^{\{\mu} k_{i}^{\nu\}}\left(\bar{\theta}_{j} \bar{\epsilon}_{j} q\right)+k_{i}^{\{\mu} k_{j}^{\nu\}}\left(\bar{\theta}_{i} \bar{\epsilon}_{i} q\right)\right)\left(q \theta_{j} \epsilon_{j}\right)}{\left|z_{i}-z_{j}\right|^{2}}\left(I_{i}^{(-1)^{i}}+I^{(-1)_{j}^{j}}\right) \\
& +\frac{1}{2}\left(\frac{\alpha^{\prime}}{2}\right)^{2} \sum_{i \neq j \neq l} \frac{k_{i}^{\{\mu} k_{i}^{\nu\}}\left(q \theta_{j} \epsilon_{j}\right)\left(q \bar{\theta}_{l} \bar{\epsilon}_{l}\right)}{\left(z_{i}-z_{j}\right)\left(\bar{z}_{i}-\bar{z}_{l}\right)} I^{(-1)^{i}}{ }_{i}^{i}+\frac{1}{2}\left(\frac{\alpha^{\prime}}{2}\right)^{2} \sum_{i \neq j \neq l} \frac{k_{i}^{\{\mu} k_{j}^{\nu\}}\left(q \theta_{l} \epsilon_{l}\right)\left(q \bar{\theta}_{l} \bar{\epsilon}_{l}\right)}{\left(z_{i}-z_{l}\right)\left(\bar{z}_{j}-\bar{z}_{l}\right)} I^{(-1)^{l}}{ }_{l}^{l} \\
& +\left(\frac{\alpha^{\prime}}{2}\right)^{2} \sum_{i \neq j \neq l} \frac{k_{i}^{\{\mu} k_{j}^{\nu\}}\left(q \theta_{l} \epsilon_{l}\right)\left(q \bar{\theta}_{i} \bar{\epsilon}_{i}\right)}{\left(z_{i}-z_{l}\right)\left(\bar{z}_{i}-\bar{z}_{j}\right)} I^{(-1)^{i}}{ }_{i}-\frac{\alpha^{\prime}}{2} \sum_{i \neq j}\left(\theta_{i} \epsilon_{i}^{\{\mu} k_{j}^{\nu\}}\left(\bar{\theta}_{i} \bar{\epsilon}_{i} q\right)+\theta_{i} \epsilon^{\{\mu} k_{i}^{\nu\}}\left(\bar{\theta}_{j} \bar{\epsilon}_{j} q\right)\right) I^{(0)}{ }_{i i}^{i j} \\
& -\frac{\alpha^{\prime}}{2} \sum_{i \neq j \neq l} \theta_{i} \epsilon_{i}^{\{\mu} k_{j}^{\nu\}}\left(q \bar{\theta}_{l} \bar{\epsilon}_{l}\right) I^{(0)}{ }_{i i}^{j l}+\sum_{i, j=1}^{n} \theta_{i} \epsilon_{i}^{\{\mu} \bar{\theta}_{j} \bar{\epsilon}_{j}^{\nu\}} I_{i i}^{(1)}{ }_{i i}^{j j}
\end{aligned}
$$

The first line, the first term of the second line, and the last term are all real, so their complex conjugate simply brings a factor of two. We may use the following identities to show that the first and second line and the first term in the third line match the second part of $S_{1}^{(2)}$ :

$$
I_{i j}^{i j}=I_{i j}^{j i}=\frac{I^{(-1)_{i}^{i}}+I^{(-1)_{j}^{j}}}{\left|z_{i}-z_{j}\right|^{2}}+O\left(q^{0}\right) ; \quad I_{i j}^{i l}=\frac{I^{(-1)}{ }_{i}^{i}}{\left(z_{i}-z_{j}\right)\left(\bar{z}_{i}-\bar{z}_{l}\right)}+O\left(q^{0}\right)
$$




$$
I_{i l}^{j l}=\frac{\left.I_{l}^{(-1)}\right)_{l}^{l}}{\left(z_{i}-z_{l}\right)\left(\bar{z}_{j}-\bar{z}_{l}\right)}+O\left(q^{0}\right) ; \quad I_{i l}^{i j}=\frac{I^{(-1)_{i}^{i}}}{\left(z_{i}-z_{l}\right)\left(\bar{z}_{i}-\bar{z}_{j}\right)}+O\left(q^{0}\right) .
$$

The last term in eq. (B.9) is trivially matched by $S_{2}^{(0)}$ and all the remaining terms are matched by the second part of $S_{3}^{(1)}$.

Next, consider

$$
A_{\theta^{3}}^{\mu \nu}=-\left(\frac{\alpha^{\prime}}{2}\right)^{\frac{3}{2}} \sum_{i \neq j \neq l} \frac{\theta_{i} \epsilon_{i}^{\{\mu}\left(q \theta_{l} \epsilon_{l}\right)\left(q \theta_{j} \epsilon_{j}\right)}{\left(z_{i}-z_{j}\right)^{2}\left(z_{j}-z_{l}\right)}\left(k_{i}^{\nu\}} I_{i}^{(-1)^{i}}-k_{j}^{\nu\}} I_{j}^{(-1)^{j}}\right)
$$

It is easily seen that this expression matches the first part of $S_{3}^{(2)}$ through order $q$, after using the identities

$$
\begin{aligned}
& I_{i i j l}^{j}=I_{i i l j}^{j}=\frac{I_{j}^{(-1)_{j}^{j}}}{\left(z_{j}-z_{l}\right)\left(z_{i}-z_{j}\right)^{2}}+O\left(q^{0}\right) \\
& I_{i i j l}^{i}=I_{i}^{(-1)^{i}}\left[-\frac{1}{\left(z_{i}-z_{j}\right)^{2}\left(z_{j}-z_{l}\right)}+\frac{1}{\left(z_{i}-z_{l}\right)^{2}\left(z_{j}-z_{l}\right)}\right]+O\left(q^{0}\right)
\end{aligned}
$$

and the property that $I_{\text {iilm }}^{j}$ only contributes at order $q^{-1}$ if either of the indices $i, l$ or $m$ equals $j$.

Next, we consider

$$
\begin{aligned}
A_{\theta^{2} \bar{\theta}}^{\mu \nu}= & \sqrt{\frac{\alpha^{\prime}}{2}} \sum_{i \neq j} \frac{\bar{\theta}_{i} \bar{\epsilon}_{i}^{\{\nu}\left(-\theta_{j} \epsilon_{j}^{\mu\}}\left(q \theta_{i} \epsilon_{i}\right)+\theta_{i} \epsilon_{i}^{\mu\}}\left(q \theta_{j} \epsilon_{j}\right)\right)}{\left(z_{i}-z_{j}\right)} I_{j i}^{(0)^{i i}}+\sqrt{\frac{\alpha^{\prime}}{2}} \sum_{i \neq j \neq l} \frac{\bar{\theta}_{j} \bar{\epsilon}_{j}^{\{\nu} \theta_{i} \epsilon_{i}^{\mu\}}\left(q \theta_{l} \epsilon_{l}\right)}{\left(z_{i}-z_{l}\right)} I^{(0)^{j} j}{ }_{i l}^{j} \\
& -\left(\frac{\alpha^{\prime}}{2}\right)^{\frac{3}{2}} \sum_{i \neq j} \theta_{i} \epsilon_{i}^{\{\mu}\left[\frac{k_{i}^{\nu\}}\left(q \bar{\theta}_{j} \epsilon_{j}\right)\left(q \theta_{j} \epsilon_{j}\right)+k_{j}^{\nu\}}\left(q \bar{\theta}_{i} \epsilon_{i}\right)\left(q \theta_{j} \epsilon_{j}\right)}{\left|z_{i}-z_{j}\right|^{2}\left(z_{i}-z_{j}\right)}\right]\left[I^{(-1)^{i}}{ }_{i}+I^{(-1)^{j}}{ }_{j}\right] \\
& -\left(\frac{\alpha^{\prime}}{2}\right)^{\frac{3}{2}} \sum_{i \neq j \neq l} \theta_{i} \epsilon^{\{\mu}\left[\frac{k_{i}^{\nu\}}\left(q \bar{\theta}_{j} \epsilon_{j}\right)\left(q \theta_{l} \epsilon_{l}\right)+k_{j}^{\nu\}}\left(q \bar{\theta}_{i} \bar{\epsilon}_{i}\right)\left(q \theta_{l} \epsilon_{l}\right)}{\left(z_{i}-z_{l}\right)^{2}\left(\bar{z}_{i}-\bar{z}_{j}\right)}\right] I^{(-1)^{i}}{ }_{i} \\
& +\left(\frac{\alpha^{\prime}}{2}\right)^{\frac{3}{2}} \sum_{i \neq j \neq l} \frac{\theta_{i} \epsilon_{i}^{\{\mu} k_{j}^{\nu\}}\left(q \bar{\theta}_{l} \bar{\epsilon}_{l}\right)\left(q \theta_{j} \epsilon_{j}\right)}{\left(\bar{z}_{j}-\bar{z}_{l}\right)\left(z_{i}-z_{j}\right)^{2}} I^{(-1)^{j}}{ }_{j}-\left(\frac{\alpha^{\prime}}{2}\right)^{\frac{3}{2}} \sum_{i \neq j \neq l} \frac{\theta_{i} \epsilon_{i}^{\{\mu} k_{j}^{\nu\}}\left(q \bar{\theta}_{l} \bar{\epsilon}_{l}\right)\left(q \theta_{l} \epsilon_{l}\right)}{\left(\bar{z}_{j}-\bar{z}_{l}\right)\left(z_{i}-z_{l}\right)^{2}} I^{(-1)^{l}}{ }_{l}^{l}
\end{aligned}
$$

The terms in the first line match $S_{2}^{(1)}$, since $I^{(0)}{ }_{j i}^{i i}\left(z_{i}-z_{j}\right)^{-1}=-I^{(0)}{ }_{i i j}^{i i}+\mathcal{O}(q)$ and $I^{(0)}{ }_{j i}^{i i}\left(z_{i}-\right.$ $\left.z_{l}\right)^{-1}=-I^{(0)^{j j}}+\mathcal{O}(q)$. For the remaining terms, all the dependence on the $z_{i}$ variables in the second line is matched by the integrals $I^{(-1)^{i j}}=I^{(-1)^{j i}}{ }_{i i j}$, while dependence on the $z_{i}$ variables in the third line, instead, matches the integrals $I^{(-1)_{i i l}^{j i}}=I^{(-1)}{ }_{i i l}^{i j}$. Finally the last two terms follow respectively from the integrals $I^{(-1)^{j l}}{ }_{i j}$ and $I^{(-1)^{l j}}$. Altogether these remaining terms thus match the second part of $S_{3}^{(2)}$.

The last expression to be considered is

$$
\begin{aligned}
A_{\theta^{2} \bar{\theta}^{2}}^{\mu \nu}= & \left(\frac{\alpha^{\prime}}{2}\right) \sum_{i \neq j} \frac{\theta_{i} \epsilon_{i}^{\{\nu}\left(q \theta_{j} \epsilon_{j}\right)}{\left|z_{i}-z_{j}\right|^{4}}\left(\bar{\theta}_{i} \epsilon_{i}^{\mu\}}\left(q \bar{\theta}_{j} \epsilon_{j}\right)-\bar{\theta}_{j} \epsilon_{j}^{\mu\}}\left(q \bar{\theta}_{i} \epsilon_{i}\right)\right)\left[I^{(-1)^{i}}+I^{(-1)^{j}}\right] \\
& +\left(\frac{\alpha^{\prime}}{2}\right) \sum_{i \neq j \neq l} \theta_{i} \epsilon_{i}^{\{\nu}\left[\frac{\left(q \theta_{j} \epsilon_{j}\right) \bar{\theta}_{i} \bar{\epsilon}_{i}^{\mu\}}\left(q \bar{\theta}_{l} \epsilon_{l}\right)}{\left(z_{i}-z_{j}\right)^{2}\left(\bar{z}_{i}-\bar{z}_{l}\right)^{2}} I_{i}^{(-1)^{i}}-\frac{\left(q \theta_{l} \epsilon_{l}\right) \bar{\theta}_{j} \bar{\epsilon}_{j}^{\mu\}}\left(q \bar{\theta}_{i} \bar{\epsilon}_{i}\right)}{\left(z_{i}-z_{l}\right)^{2}\left(\bar{z}_{i}-\bar{z}_{j}\right)^{2}} I^{(-1)^{i}}{ }_{i}\right.
\end{aligned}
$$




$$
\left.-\frac{\left(q \theta_{j} \epsilon_{j}\right) \bar{\theta}_{j} \bar{\epsilon}_{j}^{\mu\}}\left(q \bar{\theta}_{l} \bar{\epsilon}_{l}\right)}{\left(z_{i}-z_{j}\right)^{2}\left(\bar{z}_{l}-\bar{z}_{j}\right)^{2}} I^{(-1)^{j}}+\frac{\left(q \theta_{l} \epsilon_{l}\right) \bar{\theta}_{j} \bar{\epsilon}_{j}^{\mu\}}\left(q \bar{\theta}_{l} \epsilon_{l}\right)}{\left(z_{i}-z_{l}\right)^{2}\left(\bar{z}_{l}-\bar{z}_{j}\right)^{2}} I^{(-1)}{ }_{l}^{l}\right]
$$

The first line can be expressed in terms of the integrals $I^{(-1)}{ }_{i i j}^{i i j}=-I^{(-1)}{ }_{i i j}^{j j i}$, matching the similar type of terms in $S_{2}^{(2)}$. Similarly the other terms can be expressed in terms of the integrals $I_{i i j}^{(-1)}{ }_{i i l}^{i(-1)}{ }_{i i l}^{j j i}, I^{(-1)_{i i j}^{j j l}}$ and $I_{i i l}^{(-1)}$, matching the remaining types of non-vanishing terms in $S_{2}^{(2)}$.

Finally, by the same procedures as above, it is easy to check that the remaining terms to be matched in eqs. (B.5) and (B.8) are obtained by acting on the $n$-point amplitude with the operators defined in the second and third line of eq. (2.24). These operator simultaneously produce terms, which exactly cancel the unmatched terms in eqs. (B.4) and (B.6). This proves the uniqueness of the subsubleading soft operator in eq. (2.24) applicable to the graviton and the dilaton.

Open Access. This article is distributed under the terms of the Creative Commons Attribution License (CC-BY 4.0), which permits any use, distribution and reproduction in any medium, provided the original author(s) and source are credited.

\section{References}

[1] A. Strominger, Asymptotic symmetries of Yang-Mills theory, JHEP 07 (2014) 151 [arXiv: 1308.0589] [INSPIRE].

[2] A. Strominger, On BMS invariance of gravitational scattering, JHEP 07 (2014) 152 [arXiv:1312.2229] [INSPIRE].

[3] T. He, V. Lysov, P. Mitra and A. Strominger, BMS supertranslations and Weinberg's soft graviton theorem, JHEP 05 (2015) 151 [arXiv:1401.7026] [INSPIRE].

[4] T. Adamo, E. Casali and D. Skinner, Perturbative gravity at null infinity, Class. Quant. Grav. 31 (2014) 225008 [arXiv:1405.5122] [INSPIRE].

[5] Y. Geyer, A.E. Lipstein and L. Mason, Ambitwistor strings at null infinity and (subleading) soft limits, Class. Quant. Grav. 32 (2015) 055003 [arXiv:1406.1462] [INSPIRE].

[6] T. Adamo and E. Casali, Perturbative gauge theory at null infinity, Phys. Rev. D 91 (2015) 125022 [arXiv: 1504.02304] [INSPIRE].

[7] D. Kapec, V. Lysov, S. Pasterski and A. Strominger, Semiclassical Virasoro symmetry of the quantum gravity $S$-matrix, JHEP 08 (2014) 058 [arXiv: 1406.3312] [INSPIRE].

[8] T. He, P. Mitra, A.P. Porfyriadis and A. Strominger, New symmetries of massless QED, JHEP 10 (2014) 112 [arXiv:1407.3789] [InSPIRE].

[9] V. Lysov, S. Pasterski and A. Strominger, Low's subleading soft theorem as a symmetry of QED, Phys. Rev. Lett. 113 (2014) 111601 [arXiv:1407.3814] [InSPIRE].

[10] M. Campiglia and A. Laddha, Asymptotic symmetries and subleading soft graviton theorem, Phys. Rev. D 90 (2014) 124028 [arXiv:1408.2228] [INSPIRE].

[11] D. Kapec, V. Lysov and A. Strominger, Asymptotic symmetries of massless QED in even dimensions, arXiv:1412.2763 [INSPIRE]. 
[12] A. Mohd, A note on asymptotic symmetries and soft-photon theorem, JHEP 02 (2015) 060 [arXiv:1412.5365] [INSPIRE].

[13] M. Campiglia and A. Laddha, New symmetries for the gravitational S-matrix, JHEP 04 (2015) 076 [arXiv: 1502.02318] [INSPIRE].

[14] D. Kapec, V. Lysov, S. Pasterski and A. Strominger, Higher-dimensional supertranslations and Weinberg's soft graviton theorem, arXiv:1502.07644 [INSPIRE].

[15] T. He, P. Mitra and A. Strominger, 2D Kac-Moody symmetry of 4D Yang-Mills theory, arXiv: 1503.02663 [INSPIRE].

[16] M. Campiglia and A. Laddha, Asymptotic symmetries of QED and Weinberg's soft photon theorem, JHEP 07 (2015) 115 [arXiv:1505.05346] [INSPIRE].

[17] D. Kapec, M. Pate and A. Strominger, New symmetries of QED, arXiv:1506.02906 [INSPIRE].

[18] S.G. Avery and B.U.W. Schwab, Burg-Metzner-Sachs symmetry, string theory and soft theorems, Phys. Rev. D 93 (2016) 026003 [arXiv: 1506.05789] [inSPIRE].

[19] M. Campiglia and A. Laddha, Asymptotic symmetries of gravity and soft theorems for massive particles, JHEP 12 (2015) 094 [arXiv: 1509. 01406] [INSPIRE].

[20] S.G. Avery and B.U.W. Schwab, Noether's second theorem and Ward identities for gauge symmetries, JHEP 02 (2016) 031 [arXiv:1510.07038] [INSPIRE].

[21] T.T. Dumitrescu, T. He, P. Mitra and A. Strominger, Infinite-dimensional fermionic symmetry in supersymmetric gauge theories, arXiv:1511.07429 [INSPIRE].

[22] M. Mirbabayi and M. Simonović, Weinberg soft theorems from Weinberg adiabatic modes, arXiv: 1602.05196 [INSPIRE].

[23] R.H. Boels and W. Wormsbecher, Spontaneously broken conformal invariance in observables, arXiv: 1507.08162 [INSPIRE].

[24] P. Di Vecchia, R. Marotta, M. Mojaza and J. Nohle, New soft theorems for the gravity dilaton and the Nambu-Goldstone dilaton at subsubleading order, Phys. Rev. D 93 (2016) 085015 [arXiv: 1512.03316] [INSPIRE].

[25] C. Cheung, K. Kampf, J. Novotny and J. Trnka, Effective field theories from soft limits of scattering amplitudes, Phys. Rev. Lett. 114 (2015) 221602 [arXiv:1412.4095] [INSPIRE].

[26] H. Lüo and C. Wen, Recursion relations from soft theorems, JHEP 03 (2016) 088 [arXiv: 1512.06801] [INSPIRE].

[27] F.E. Low, Scattering of light of very low frequency by systems of spin 1/2, Phys. Rev. 96 (1954) 1428 [INSPIRE].

[28] M. Gell-Mann and M.L. Goldberger, Scattering of low-energy photons by particles of spin 1/2, Phys. Rev. 96 (1954) 1433 [InSPIRE].

[29] F.E. Low, Bremsstrahlung of very low-energy quanta in elementary particle collisions, Phys. Rev. 110 (1958) 974 [INSPIRE].

[30] S. Saito, Low-energy theorem for Compton scattering, Phys. Rev. 184 (1969) 1894 [INSPIRE].

[31] T.H. Burnett and N.M. Kroll, Extension of the low soft photon theorem, Phys. Rev. Lett. 20 (1968) 86 [INSPIRE]. 
[32] J.S. Bell and R. Van Royen, On the Low-Burnett-Kroll theorem for soft-photon emission, Nuovo Cim. A 60 (1969) 62 [InSPIRE].

[33] V. Del Duca, High-energy bremsstrahlung theorems for soft photons, Nucl. Phys. B 345 (1990) 369 [INSPIRE].

[34] S. Weinberg, Photons and gravitons in S-matrix theory: derivation of charge conservation and equality of gravitational and inertial mass, Phys. Rev. 135 (1964) B1049.

[35] S. Weinberg, Infrared photons and gravitons, Phys. Rev. 140 (1965) B516.

[36] D.J. Gross and R. Jackiw, Low-energy theorem for graviton scattering, Phys. Rev. 166 (1968) 1287 [INSPIRE].

[37] R. Jackiw, Low-energy theorems for massless bosons: photons and gravitons, Phys. Rev. 168 (1968) 1623 [INSPIRE].

[38] E. Laenen, G. Stavenga and C.D. White, Path integral approach to eikonal and next-to-eikonal exponentiation, JHEP 03 (2009) 054 [arXiv:0811.2067] [INSPIRE].

[39] E. Laenen, L. Magnea, G. Stavenga and C.D. White, Next-to-eikonal corrections to soft gluon radiation: a diagrammatic approach, JHEP 01 (2011) 141 [arXiv:1010.1860] [INSPIRE].

[40] C.D. White, Factorization properties of soft graviton amplitudes, JHEP 05 (2011) 060 [arXiv:1103.2981] [INSPIRE].

[41] F. Cachazo and A. Strominger, Evidence for a new soft graviton theorem, arXiv:1404.4091 [INSPIRE].

[42] E. Casali, Soft sub-leading divergences in Yang-Mills amplitudes, JHEP 08 (2014) 077 [arXiv: 1404.5551] [INSPIRE].

[43] B.U.W. Schwab and A. Volovich, Subleading soft theorem in arbitrary dimensions from scattering equations, Phys. Rev. Lett. 113 (2014) 101601 [arXiv:1404.7749] [INSPIRE].

[44] Z. Bern, S. Davies and J. Nohle, On loop corrections to subleading soft behavior of gluons and gravitons, Phys. Rev. D 90 (2014) 085015 [arXiv:1405.1015] [INSPIRE].

[45] S. He, Y.-T. Huang and C. Wen, Loop corrections to soft theorems in gauge theories and gravity, JHEP 12 (2014) 115 [arXiv:1405.1410] [INSPIRE].

[46] A.J. Larkoski, Conformal invariance of the subleading soft theorem in gauge theory, Phys. Rev. D 90 (2014) 087701 [arXiv: 1405.2346] [INSPIRE].

[47] F. Cachazo and E.Y. Yuan, Are soft theorems renormalized?, arXiv:1405.3413 [INSPIRE].

[48] N. Afkhami-Jeddi, Soft graviton theorem in arbitrary dimensions, arXiv:1405.3533 [INSPIRE].

[49] J. Broedel, M. de Leeuw, J. Plefka and M. Rosso, Constraining subleading soft gluon and graviton theorems, Phys. Rev. D 90 (2014) 065024 [arXiv:1406.6574] [INSPIRE].

[50] C.D. White, Diagrammatic insights into next-to-soft corrections, Phys. Lett. B 737 (2014) 216 [arXiv: 1406.7184] [INSPIRE].

[51] M. Zlotnikov, Sub-sub-leading soft-graviton theorem in arbitrary dimension, JHEP 10 (2014) 148 [arXiv: 1407.5936] [INSPIRE].

[52] C. Kalousios and F. Rojas, Next to subleading soft-graviton theorem in arbitrary dimensions, JHEP 01 (2015) 107 [arXiv: 1407.5982] [INSPIRE]. 
[53] Y.-J. Du, B. Feng, C.-H. Fu and Y. Wang, Note on soft graviton theorem by KLT relation, JHEP 11 (2014) 090 [arXiv: 1408.4179] [INSPIRE].

[54] D. Bonocore, E. Laenen, L. Magnea, L. Vernazza and C.D. White, The method of regions and next-to-soft corrections in Drell-Yan production, Phys. Lett. B 742 (2015) 375 [arXiv: 1410.6406] [INSPIRE].

[55] H. Lüo, P. Mastrolia and W.J. Torres Bobadilla, Subleading soft behavior of QCD amplitudes, Phys. Rev. D 91 (2015) 065018 [arXiv:1411.1669] [inSPIRE].

[56] J. Broedel, M. de Leeuw, J. Plefka and M. Rosso, Local contributions to factorized soft graviton theorems at loop level, Phys. Lett. B 746 (2015) 293 [arXiv:1411.2230] [INSPIRE].

[57] A.J. Larkoski, D. Neill and I.W. Stewart, Soft theorems from effective field theory, JHEP 06 (2015) 077 [arXiv: 1412.3108] [INSPIRE].

[58] A. Sabio Vera and M.A. Vazquez-Mozo, The double copy structure of soft gravitons, JHEP 03 (2015) 070 [arXiv: 1412.3699] [INSPIRE].

[59] A.E. Lipstein, Soft theorems from conformal field theory, JHEP 06 (2015) 166 [arXiv: 1504.01364] [INSPIRE].

[60] S.D. Alston, D.C. Dunbar and W.B. Perkins, n-point amplitudes with a single negative-helicity graviton, Phys. Rev. D 92 (2015) 065024 [arXiv:1507.08882] [INSPIRE].

[61] J. Rao and B. Feng, Note on identities inspired by new soft theorems, JHEP 04 (2016) 173 [arXiv: 1604.00650] [INSPIRE].

[62] Z. Bern, S. Davies, P. Di Vecchia and J. Nohle, Low-energy behavior of gluons and gravitons from gauge invariance, Phys. Rev. D 90 (2014) 084035 [arXiv:1406.6987] [InSPIRE].

[63] M. Bianchi, S. He, Y.-T. Huang and C. Wen, More on soft theorems: trees, loops and strings, Phys. Rev. D 92 (2015) 065022 [arXiv:1406.5155] [InSPIRE].

[64] Z.-W. Liu, Soft theorems in maximally supersymmetric theories, Eur. Phys. J. C 75 (2015) 105 [arXiv: 1410.1616] [INSPIRE].

[65] J. Rao, Soft theorem of $N=4$ SYM in Grassmannian formulation, JHEP 02 (2015) 087 [arXiv: 1410.5047] [INSPIRE].

[66] W.-M. Chen, Y.-T. Huang and C. Wen, New fermionic soft theorems for supergravity amplitudes, Phys. Rev. Lett. 115 (2015) 021603 [arXiv: 1412.1809] [INSPIRE].

[67] W.-M. Chen, Y.-T. Huang and C. Wen, From U(1) to E : $_{8}$ soft theorems in supergravity amplitudes, JHEP 03 (2015) 150 [arXiv:1412.1811] [INSPIRE].

[68] L.V. Bork and A.I. Onishchenko, On soft theorems and form factors in $N=4 S Y M$ theory, JHEP 12 (2015) 030 [arXiv:1506.07551] [INSPIRE].

[69] S. Chin, S. Lee and Y. Yun, ABJM amplitudes in U-gauge and a soft theorem, JHEP 11 (2015) 088 [arXiv: 1508.07975] [INSPIRE].

[70] A. Brandhuber, E. Hughes, B. Spence and G. Travaglini, One-loop soft theorems via dual superconformal symmetry, JHEP 03 (2016) 084 [arXiv:1511.06716] [INSPIRE].

[71] S.G. Avery and B.U.W. Schwab, Residual local supersymmetry and the soft gravitino, Phys. Rev. Lett. 116 (2016) 171601 [arXiv: 1512.02657] [INSPIRE].

[72] V. Lysov, Asymptotic fermionic symmetry from soft gravitino theorem, arXiv:1512.03015 [INSPIRE]. 
[73] B.U.W. Schwab, Subleading soft factor for string disk amplitudes, JHEP 08 (2014) 062 [arXiv: 1406.4172] [INSPIRE].

[74] B.U.W. Schwab, A note on soft factors for closed string scattering, JHEP 03 (2015) 140 [arXiv:1411.6661] [INSPIRE].

[75] M. Bianchi and A.L. Guerrieri, On the soft limit of open string disk amplitudes with massive states, JHEP 09 (2015) 164 [arXiv: 1505.05854] [INSPIRE].

[76] A.L. Guerrieri, Soft behavior of string amplitudes with external massive states, in IFAE 2015, Rome Italy April 8-10 2015 [arXiv: 1507.08829] [INSPIRE].

[77] P. Di Vecchia, R. Marotta and M. Mojaza, Soft theorems from string theory, Fortsch. Phys. 64 (2016) 389 [arXiv:1511.04921] [INSPIRE].

[78] P. Di Vecchia, R. Marotta and M. Mojaza, Soft theorem for the graviton, dilaton and the Kalb-Ramond field in the bosonic string, JHEP 05 (2015) 137 [arXiv: 1502.05258] [INSPIRE].

[79] F. Cachazo, S. He and E.Y. Yuan, New double soft emission theorems, Phys. Rev. D 92 (2015) 065030 [arXiv: 1503.04816] [INSPIRE].

[80] T. Klose, T. McLoughlin, D. Nandan, J. Plefka and G. Travaglini, Double-soft limits of gluons and gravitons, JHEP 07 (2015) 135 [arXiv: 1504.05558] [INSPIRE].

[81] A. Volovich, C. Wen and M. Zlotnikov, Double soft theorems in gauge and string theories, JHEP 07 (2015) 095 [arXiv: 1504.05559] [INSPIRE].

[82] Y.-J. Du and H. Lüo, On single and double soft behaviors in NLSM, JHEP 08 (2015) 058 [arXiv: 1505.04411] [INSPIRE].

[83] P. Di Vecchia, R. Marotta and M. Mojaza, Double-soft behavior for scalars and gluons from string theory, JHEP 12 (2015) 150 [arXiv:1507.00938] [INSPIRE].

[84] I. Low, Double soft theorems and shift symmetry in nonlinear $\sigma$-models, Phys. Rev. D 93 (2016) 045032 [arXiv: 1512.01232] [INSPIRE].

[85] N. Arkani-Hamed, F. Cachazo and J. Kaplan, What is the simplest quantum field theory?, JHEP 09 (2010) 016 [arXiv:0808.1446] [INSPIRE].

[86] M. Ademollo et al., Soft dilations and scale renormalization in dual theories, Nucl. Phys. B 94 (1975) 221 [INSPIRE].

[87] J.A. Shapiro, On the renormalization of dual models, Phys. Rev. D 11 (1975) 2937 [INSPIRE].

[88] T. Yoneya, String coupling constant and dilaton vacuum expectation value in string field theory, Phys. Lett. B 197 (1987) 76 [InSPIRE].

[89] H. Hata, Soft dilaton theorem in string field theory, Prog. Theor. Phys. 88 (1992) 1197 [INSPIRE].

[90] R.R. Metsaev and A.A. Tseytlin, Order $\alpha^{\prime}$ (two loop) equivalence of the string equations of motion and the $\sigma$-model Weyl invariance conditions: dependence on the dilaton and the antisymmetric tensor, Nucl. Phys. B 293 (1987) 385 [INSPIRE].

[91] M. Bianchi and A.L. Guerrieri, On the soft limit of closed string amplitudes with massive states, Nucl. Phys. B 905 (2016) 188 [arXiv:1512.00803] [INSPIRE].

[92] B. Zwiebach, Curvature squared terms and string theories, Phys. Lett. B 156 (1985) 315 [INSPIRE]. 